\title{
Intelligence vs. Artificial Intelligence: The King Is Naked
}

\author{
Claudio Messori \\ Str. Villaggio Prinzera 1, Fraz. Boschi di Bardone, Terenzo, Italy \\ Email: messori.claudio@gmail.com
}

How to cite this paper: Messori, C. (2016) Intelligence vs. Artificial Intelligence: The King Is Naked. Open Access Library Journal, 3: e3115.

http://dx.doi.org/10.4236/oalib.1103115

Received: October 6, 2016

Accepted: November 11, 2016

Published: November 14, 2016

Copyright $(2016$ by author and Open Access Library Inc.

This work is licensed under the Creative Commons Attribution International License (CC BY 4.0).

http://creativecommons.org/licenses/by/4.0/

(c) (i) Open Access

\section{Abstract}

The sociological dimension of Artificial Intelligence (AI), and automation, is placed in the path traced by the man-machine integration process, started in the eighteenth century by the Industrial Revolution that assumes its current connotation after World War II. The use of the term intelligence, which appears in the expression $\mathrm{Ar}$ tificial Intelligence, is shown to be improper and misleading, and the expression itself should be replaced, eventually, by Artificial Simulation of the Savant Syndrome. By adopting a theoretical perspective, namely Endo-Dynamo-Tensive Model [Messori 2012B], it is traced the mapping of human's neurological (neuro-dynamics) and psychological (psycho-dynamics) dimension, and is provided the coordinates to be followed in the phenomenological qualification and definition of the psychic-mentalcognitive function intelligence. The neuro-dynamics of the Nervous System (NS), is taken into account by adopting the theoretical, interpretative and investigative perspective indicated by the particular line of research developed in the context of Quantum Field Theory (QFT) and QED (Quantum Electrodynamic Field Theory), that describes the water of which all living systems are composed, i.e. biological water, as water in a coherent oscillatory phase or state other than that of common water, named super-coherent oscillatory state. By introducing the possible functional role exerted within brain activity by glial cells, cerebrospinal fluid, intra- and extracellular fluid is outlined the overcoming of the classical neuroscience paradigm, based on the vision of brain activity as ruled by networks of neurons interconnected by synapses. The body-mind hard problem is taken into account and a solution is advanced. The psycho-dynamics of the humans mind territory is taken into account according to the four poles of mental functions introduced by C.G. Jung in his $P s y$ chological Types (1921), where he introduces a hierarchy of mental functions in two mental bipolar dimensions (dichotomies). These are sensing (attentiveness by means of the sense organs) coupled to intuition (awareness in unconscious way or being aware of unconscious contents) and thinking (function of intellectual cognition; the forming of logical conclusions) coupled to feeling (function of subjective estimation). 
At the conclusion of this work, it is provided the phenomenological definition of intelligence that does not contemplate the possibility to apply to neuroscience, and to natural and human sciences in general, the paradigm that inspires the research on AI, i.e. computational model and Information Theory.

\section{Subject Areas}

Anthropology

\section{Keywords}

Artificial Intelligence, Man-Machine Integration Process, Artificial Simulation of the Savant Syndrome, Endo-Dynamo-Tensive Model, Biological Water, Autopoietic Psycho-Neuro Dynamics, Intelligence

\section{Artificial Intelligence: Falsifying the Identity of the Mental Phenomenon and Its Functions}

Groucho Marx said that "Military intelligence is a contradiction in terms". Well, AI is the hyper-technological product of this contradiction, and I guess

Norbert Wiener was right in saying that

“... wartime technology, which has furnished

from small bits of scientific truth great warehouses of potential threat and arsenals of destruction, has much to thank scientist for their intellectual prostitution has been significant".

Anonymous

To possess and exert control is the mantra of the third millennium, and the computerization of society meets this purpose: "The computerization of society... has essentially been a side effect of the computerization of war". (Frank Rose). The globalization of computerization is a project planned by the US military fields during the years of the Cold War ${ }^{1}$, which offers to those who run it the possibility to control almost everything based on telecommunications and on computer processes. The Artificial Intelligence (AI) is strategically located in this project.

Artificial Intelligence, a term coined in 1956 by the young American mathematician John McCarthy, ranks in the footsteps of the man-machine integration process started by the eighteenth-century Industrial Revolution, and is the legitimate daughter of the Information Theory, a discipline born in the Telecommunications field (telegraph and telephone) between the 20's and 30's and developed between the 40's and 50's separately by Claude Shannon (The Mathematical Theory of Communication) and Norbert Wiener (Cybernetics). The term intelligence that appears in the expression coined in 1956 by McCarthy is a legacy of the terminology used by one of the pioneers of Infor-

${ }^{1}$ See: Kay, L.E. (2000) Who Wrote the Book of Life? A History of the Genetic Code. Stanford University Press, Stanford. 
mation Theory, Harry Nyquist of the American Telephone and Telegraph Company (AT \& T). In 1924 Nyquist publishes an article on the Bell System Technical Journal (BSTJ) entitled Certain Factors Affecting Telegraph Speed, where deals with the factors that affect the "maximum speed of transmission of intelligence". For those in the works of the period, the metaphorical term "intelligence" used by Nyquist for the transmission of an electromagnetic signal, appeared improper and misleading, compromised by anthropomorphic and psychological references incompatible with the subject matter. The transmission of signals between machines (coder/decoder), i.e. the sending and receiving of electromagnetic state variations through a medium (broadcast or via cable), could not be in any way confused with the transmission of meaning (messages) and neither could be associated with human properties generated by the possibility to feel, to sense, to think, to perceive, to intuit, to hypothesize, to imagine, to invent, to reflect, to dream, to experience. Four years after the publication of Nyquist's article, a colleague of Bell Telephone Laboratories Inc., Ralph V.L. Hartley, published an article on the BSTJ, entitled Transmission of Information, where the intelligence metaphor is replaced, for reasons of "physical as contrasted with psychological considerations", by the metaphor information.

But what did Nyquist mean by the term intelligence? In fact nothing would have to deal with human properties as those listed above, or could attribute to a signal a semantic meaning. By the term intelligence, Nyquist refers to the statistically determined and decipherable component of a random signal (i.e. to the data inputs, conveyed and made available by an analogic signal, qualified by Nyquist as "the number of characters, representing different letters, figures, etc." transmitted in a certain period of time), whose transmissibility (with no loss of intelligence i.e. data) from a transmitting device (coder) to a receiver (decoder) depends on the degree of uncertainty resolution (associated with the noise inherently generated by the transmission medium or by the involved apparatuses) obtained in the signal transmission.

But times change, and what in the 20's for reasons of "physical as contrasted with psychological considerations" could not be described as intelligent, it became in the 50 's.

From the 50's onwards, a wide array of scientists, researchers, scholars and experts, who for various reasons and to varying degrees have conformed to AI, have started adopting the computational model on which AI is based, transferring it to biology, genetics, psychology, psychiatry, anthropology, ethology, neuroscience, so as to make the mental phenomenon (the res cogitans of Cartesian memory) somehow objective (according to the Cartesian dichotomy res cogitans is a fictitious, not objective entity, and as such of no interest to science), a phenomenon that ever since is governed by the same laws that regulate the transmission of signals fitted with meaning in telecommunications (coder and decoder exchange messages $\rightarrow$ cells also exchange messages) and in computer programming (computer coding is based on computation $\rightarrow$ genetic coding also is based on computation) is no longer as fictitious as it seemed. In doing so, the in vivo reality has been forged in the image and likeness of the in vitro Information 
Theory.

Unconcerned of the fact that ".the brain is not a bundle of neurons dissected in a laboratory but exists within an organism which is essentially carrying on its own auto-regulation, its nutrition and self-preservation, which feels hunger and thirst and needs social relations, while continually permeated, interwoven, by emotions, sentiments, needs, desires"', these AI followers insist on locating the brain functions in this or that area and neuronal network, in this or that sub-cellular (e.g. microtubules) or inter-cellular (e.g. synapses) structure, in this or that sequence (code), deterministic or probabilistic, of information, and not pleased with that come to resurrect the adultmetric vision of some pseudo-scientific tradition of the past, according to which ".the human infant is a decerebrated being equipped with reflexes". (Paul Emil Flechsig, 1847-1929), and "The Negro brain possesses a spinal cord of the type found in children and women, and beyond this, approaches the type of brain found in higher apes" (Emil Huschke, 1797-1858), to discredit the cognitive level of a child, associating his IQ not anymore to that of a de-cerebrated being or to that of a higher ape, but to that of a computer, alias intelligent machine.

That's what has been done in 2013, for example, by an AI experts team of the University of Illinois at Chicago (UIC), which has designed and conducted a study ${ }^{3}$ the purpose of which was to evaluate the degree of "intelligence" of the super computer ConceptNet 4, by administering to the machine a part of a well known IQ test, administered as IQ test of children aged between two years and six months and seven years and seven months, the Wechsler Preschool and Primary Scale of Intelligence Test (WPPSI) created in 1967 by American psychologist David Wechsler, already creator of the Wechsler Intelligence Scale for Children (WISC, 1949) and of the Wechsler Adult Intelligence Scale (WAIS, 1955). The study suggested that ConceptNet 4 would have an IQ comparable to that of a four-year age child.

Studies like this demonstrate two things, the first is that even in the twenty-first century kids are being targeted by new detractors of their intelligence, and the second is that the temptation to falsify the physical identity of the mental phenomenon, and its functions including intelligence, trying to pass the camel intelligence through the eye of the needle of telecommunications, information technology and cybernetics is too high so as not to bear fruits.

The definition of intelligence provided by David Wechsler goes exactly in this direction: Intelligence is the aggregate or global capacity of the individual to act purposefully, to think rationally and to deal effectively with his environment (Wechsler, 1944).

Act intentionally, think rationally, effectively deal with the environment: are these the conditions that qualify intelligence and make a being intelligent?

Intentionality and the use of rational thinking for adaptive and supra-adaptive purposes, are very late events in the history of mankind [1]. The occurrence of a behavior

${ }^{2}$ Benvenuto, S. (2002) Consciousness in the Neurosciences. In: A conversation of Sergio Benvenuto with Francisco Varela. http://www.psychomedia.it/jep/number14/varela.htm

${ }^{3}$ University of Illinois at Chicago (2013) Computer smart as a 4-year-old.

http://news.uic.edu/a-computer-as-smart-as-a-four-year-old 
clearly moved by intentionality dates back to the Middle Paleolithic, while for rational thinking strictu sensu we must wait for the Upper Paleolithic: does it means that before that, human communities were deprived of intelligence? The relational life of a two year old child is governed by the symbolic way of thinking and if left to himself will hardly be able to survive: can we consider him or her intelligent? The magical thinking that characterizes the social life of an indigenous belonging to a settled community of the Amazon forests, which for generations has never abandoned his ancestral territory, and has never changed his own traditions and his own means of livelihood, makes him an intelligent being? ConceptNet 4 can really be considered somehow intelligent?

Claim to measure intelligence with a test means to apply to the res cogitans the same reductionist and mechanistic pattern applied by the positivist paradigm to the res extensa: the structuring of the objects and phenomena that we observe inside and outside of us, happens thanks to a sequence of combinations (factorizable), guided by a code (algorithmic) among certain structural elements (building blocks). In the case of a material object, the structural elements can be molecules, atoms, particles. In the case of a fictitious object as intelligence can be, e.g. the logical-mathematical intelligence, the verbal i., the spatial $i$., the musical $i$., the kinesthesic $i$, the emotional $i$. (Howard Gardner), in turn decomposable into sub-groups, and so on and so forth. Once broken down into objectifiable structural elements, the object shall be subjected to measuring and eventually reproduced and controlled.

The result is a puzzle composed by many elements, connected to joint, in a frame which develops a given function. But in the in vivo reality things are very different, and it is not the structure that generates the function, while the opposite is true.

\subsection{Artificial Intelligence: The Man-Machine Integration Process}

If the seventeenth and early eighteenth centuries are the age of clocks, and the later eighteenth and the nineteenth centuries constitute the age of steam engines, the present time is the age of communication and control.

Norbert Wiener

The profile provided today of the res cogitans, must be functional to the modern application of the teaching imparted to history by the eighteenth-century Industrial Revolution: thanks to the positivist machines, invented and realized with the ingenuosity and the hands of skilled artisans and the money of daring patrons of the eighteenth and nineteenth-century bourgeois urban class, open up perspectives for profit and wellbeing (but also of destruction and death, the other side of the coin) unthinkable without them, a true technological Eldorado. The entire socio-economic system of almost the entire planet would not exist without the introduction of mechanical systems in the production cycle. The very idea of the production cycle belongs to the man-machine integration process, started in the eighteenth century in the workplaces, that following an entrepreneurial logic puts the human capital (the workforce) in a position of inferiority and subordination to the technological capital (technicians, machines, wea- 
pons). When patrons became entrepreneurs and artisans technicians, science and scientists became an essential part of this man-machine integration process. AI belongs, in fact and in law, to it.

Over the lifetime of a septuagenarian born in the late nineteenth century, Western society was driven by an unshakable faith in the power of science and technology, and technology itself was transformed from a haphazard process of invention to a sciencebased discipline. Steam engines and railroads, electric lights and airplanes, the telegraph, typewriter, motion pictures, radio communication, radar systems and plastics were just some of the achievements reached by the Western scientific and technological miracle between late nineteenth and the mid-twentieth, when by conceiving, building and using the first weapons of mass destruction (e.g. rockets, atomic bomb, hydrogen bomb, intercontinental ballistic missiles), the miracle showed to history its terrible destructive power.

Between the 50's and 70's, in the shadow of the devastating effects produced by the launching of the atomic bomb on Hiroshima (Little Boy) and Nagasaki (Fat Man), the machines definitely come out of crafts and industrial productive perimeter to become television sets and household appliances, partners that bring advantages and comfort in the homes' habitat of millions of people, integral part of their lives and relationship dynamics, differentiating themselves according to the consumers tastes and expectations, to set up the conditions for what will become a gradual addiction to the availability of technological solutions, more and more disconnected from the real needs of their users and increasingly self-referential.

The years between the 60's and 90's mark a new qualitative leap in the man-machine integration process, a new technological and psychological revolution that thanks to the fortunate combination between personal computers, World Wilde Web and the scion AI, whose mission is "designing systems that exhibit the characteristics associated with human intelligence, like understanding language, learning, reasoning, solving problems, and so on", and whose belief is that "every aspect of learning or any other feature of intelligence can in principle be so precisely described that a machine can be made to simulate it', transforms the machine in a metacognitive tool, in an interactive platform that can assist you in work, study, communication, socialization, in leisure, entertainment, in portal to have access to a virtual ocean where you can draw everything and its opposite. From now on, the machine is no longer just part of our habitat, the machine becomes part of us and we part of it. It is in this period of time that those who deal with AI mature the conviction that to perform the spell to animate the machine and make it as one of us, to make the illusion of its presumed intelligence the more likely, it needs to be equipped by the ability to speak, and this is one of the task of the Strategic Computing Program (SCP).

In the 90's the know-how gained during the 80 's by computer science, cybernetics, robotics and AI research, contributed to the realization of a project, the SCP, financed with funds both public (from the omnipresent American federal agency Defense Advanced Research Projects Agency, DARPA) and private (from investors as the industrial giant IBM, the Dragon Systems company, the BBN, i.e. Bolt Beranek and Newman, 
and the Systems Development Corporation, SDC), thanks to which will come out the so-called intelligent machines, namely: machine with advanced intelligence technology and high-performance computing, including speech recognition and understanding, natural-language computer interfaces, vision comprehension systems, and advanced expert systems development, provided by a significant increasing in computer performance, through parallel-computer architectures, software, and supporting microelectronics.

\subsection{Artificial Intelligence: Artificial Simulation of the Savant Syndrome Leading to Cyborgs}

The emperor walked beneath the beautiful canopy in the procession, and all the people in the street and in their windows said, "Goodness, the emperor's new clothes are incomparable! What a beautiful train on his jacket. What a perfect fit?' No one wanted it to be noticed that he could see nothing, for then it would be said that he was unfit for his position or that he was stupid. None of the emperor's clothes had ever before received such praise.

"But he doesn't have anything on?" said a small child. Hans Christian Andersen (The Emperor's New Clothes)

Drones remotely controlled via Advanced Drone Interfacing System. Anthropomorphic robots interfaced in the Cloud Machine. Vocal assistants interconnected in the cloud environment supported by a neural net...

The dawn of the third millennium marks the spread on a global scale of very performing computing devices for home, medical, scientific, military, industrial use, that thanks to sophisticated voice recognition and production systems make them able to entertain, actively and in real time, an exchange of verbal information (although virtual, because you are anyway talking to a computing platform masquerade as human interlocutor), computationally itemized and statistically endowed with meaning. A fact equipped with enormous evocative implications (fascination).

In giving voice to a machine, reemerges the magic power assigned to the word-sound by the mid and late Paleolithic human communities [1] [2] [3], subsequently merged in the post-Neolithic metaphysical and philosophical concept of psyche, pneuma, breath, ruah, prana, anima/animus, and finally in that of Energy (energheia), i.e. the ability attributed to the acoustic vibration to animate, to give life to the inanimate (a skill of which the male gender was soon made depositary, imposing the semantic baptism as an act of legitimation of reality, to be able to contend and to pull out from the female uterus her natural power to generate life).

The scientist who gives voice to a machine is like the shaman who gives voice to the spirits: the spirits speak through the shaman's intercession, machines speak through scientist intercession. The task of the Neolithic shaman (and after him of any other person appointed to act as an intermediary, medium, with the otherness) was to curry favor with the spirits, so that they could influence the forces of Nature, for the good (or for bad, black magic vs. white magic) of the community. The task of the contemporary 
shaman-scientist is much more ambitious: he wants literally subdue and even replace the forces of Nature. Meanwhile he succeeded, thanks to artificial insemination in vitro, to pull out from the female uterus (and not just thanks to the speech monopoly, but in deeds, artificially) the natural power of generating life belonging to woman.

Here, in this bacchanal that involves talking machines and a wide array of scientists, researchers, scholars and experts, who for various reasons and to varying degrees have conformed to the AI Eldorado, makes its way the idea of a future populated by intelligent machines equipped with consciousness (androids), perhaps in service to humanity or maybe not and maybe, as some claim (transhumanists), smarter, more reliable and better performing (Ray Kurzweil, chief engineer of Google, theorizes the overcoming of human intelligence by computers), definitely more programmable and controllable than humans.

If we'll have to compete with more and more "advanced generations of machines", somehow equipped with consciousness, a question arise: is there any kind of intelligence in AI, or to pursue the development of human-machine hybrids, imagining them as a first step towards the production of artificial humanoids, somehow better than humans, is a project that resuscitates eugenics in an AI format?

60 years have passed since John McCarthy coined the term Artificial Intelligence and what we can say about the research in the AI field is that: is conducted by a range of scientists and technologists with varying perspectives, interests, and motivations. Scientists tend to be interested in understanding the underlying basis of intelligence and cognition, some with an emphasis on unraveling the mysteries of human thought and others examining intelligence more broadly. Engineering-oriented researchers, by contrast, are interested in building systems that behave intelligently. Some attempt to build systems using techniques analogous to those used by humans, whereas others apply a range of techniques adopted from fields such as information theory, electrical engineering, statistics, and pattern recognition. Those in the latter category often do not necessarily consider themselves AI researchers, but rather fall into a broader category of researchers interested in machine intelligence ${ }^{4}$.

Is there any kind of intelligence in $\mathrm{AI}$ ?

In the best case, the performances of the so-called intelligent machines are and will remain a computational, artificial simulation of some abilities manifested by people affected by the savant syndrome, or syndrome of the "idiot savant", where idiot is referred to a male (savant syndrome occurs approximately four times more frequently in males than females as does the incidence of autism) suffering from a series of more or

${ }^{4}$ Hughes, T., et al. (1999) Developments in Artificial Intelligence. In: Funding a Revolution. Government Support for Computing Research, Computer Science and Telecommunications Board, National Research Council, Chapter 9, 198-225. http://www.nap.edu/read/6323/chapter/11\#199

${ }^{5}$ Savant syndrome is a rare but remarkable condition in which persons with some underlying developmental disability or other brain trauma or disorder have some extraordinary special abilities- "islands of genius" which stand in stark contrast to overall limitations. Typically these special abilities are in music, art, mathematics or mechanical/visual-spatial areas. Whatever, the special skill it is always combined with massive memory. Sometimes, but not always, the underlying disability is autism. Savant syndrome provides a unique window into the brain for the study not only of brain structure, savant skills and memory, but it also provides unique glimpses into intelligence, learning and creativity. 
less severe cognitive and mental disabilities, but that has one or more than one superdeveloped abilities, a jarring juxtaposition of ability and disability in the same person always combined with massive memory (typically eidetic memory). This is all that an intelligent machine can and will aspire to do: artificial simulation of the savant syndrome (ASSS). What the ASSS experts can do and will be able to do is to develop and get the most out of the super-memory (storing) and computational skills that qualify an intelligent machine, inventing new algorithmic systems, new software and new media devices for new applications (e.g., via emotion modelling [4] the machine will be programmed and tuned on the affective history, memories and aspirations, disappointments and expectations, desires and frustrations of their owner, so to satisfy at best their wishes and demands).

Realize an intelligent machine equipped with consciousness? Ridiculous. What can be done, and that is already being done, is to acquire new persuasive and pervasive technological tools, including robotic and computer systems exploiting AI, to be used in the military and civilian field, as a means for expansion and control of the market dynamics, or as a deterrent for the resolution of conflicts related to it, both locally and globally, on a small and large scale.

What is already being done is to prepare next generations of consumers willing, or predisposed, to become or to be transformed into one of the two types of cyborgs currently proposed by the high-tech communication marketing, the standard-integrated and the extra-integrated cyborg.

The standard-integrated cyborg (SIC) is the average technology products consumer affected by Technology Addiction or Communication Addiction, that thanks to a device inserted a dimora in his own body, a nanorobot or an intracranial microchip for neural interactions (brain-computer interfaces) for example [5], or a subcutaneous chip (e.g. transponder implantation), can interface directly, via Near Field Communication (NFC) or via Far Field Communication (FFC) [6], with an external device (e.g. a smartphone or a computer or any other wireless or bluetooth device equipped with an appropriate interface), or with the cloud (exposing himself to the risk of hacking).

This SIC will be easily identifiable and traceable and will be able to make some regular transactions, such as paying the bill at a supermarket or restaurant, make a withdrawal at an ATM, or punch the clock at entry and exit at workplace or school.

The extra-integrated cyborg (EIC), instead, is a man-machine hybrid trained to face high-risk experiences (e.g. military, policing or espionage missions, terrorist or criminal actions, space travels and stays in extra-terrestrial environments), supplemented by internal technological components which increase the self-adjusting body-mind functions (such as tolerance to pain, hunger and thirst, heat regulation, etc.), enhance the so-called normal performances (such as the ability to calculate, memorization and perceptual skills), make him or her remotely controllable and programmable [7] [8] [9] [10] [11].

This is all that can be done and that is already being done, towards some next generations of technology products consumers affected by AI Addiction, a future littered with AI applications aimed at enhancing or inhibit (drugs-like?) certain functions or skills, in order to make the subject better performing and more competitive, or simply 
more monitorable.

\section{Approaching Intelligence}

\section{The pendulum of the mind oscillates between sense and nonsense, not between right and wrong. C.G. Jung}

Intelligence is a psychic, a mental or a cognitive function? In literature these three adjectives, psychic-mental-cognitive, are often used as synonyms, but in agreement with the interpretative model of the mental phenomenon which I have set in [1] [12]-[17], it is appropriate to give them a distinct meaning. The reasons justifying the opportunity to introduce this distinction are three:

1) The first stems from the need to place the functions, in this case intelligence, in a phylogenetic perspective ${ }^{6}$, trying to identify what can be the pre-constitutive psychological requirements, essential, predisposed ante rem, innate, phylogenetically prescribed, ready to use, that underpin the capacity, the possibility and the relational trend of the genus Homo, and on what structural and functional transformations and solutions, they went to meet during the long process of in-formation of the inner life (insight) of human communities.

2) The second stems from the need to determine how these transformations and solutions have affected, over a period of time ranging from the Lower Paleolithic to the Upper Paleolithic, on the information of the functions.

3) The third stems from the need to clarify how and to what extent these functional transformations and solutions are correlated to the structural transformations and solutions that has faced the neuro-anatomy and/or the neuro-biology and/or the neurophysiology of the CNS. In particular, we would like to clarify whether and to what extent there is a clearly established correspondence between the increase in functional capacities (e.g. Intelligence) and the increase of the cerebral mass in relation to body mass and/or the increase of the density of neurons and/or synaptic junctions in relation to the cerebral mass.

These three reasons lay down two distinct perspectives of investigation of the function. The first aims to explore the dynamics of the inner life of the genus Homo, that is its psycho-dynamics (the constitutive patterns, the trans-generational and supra-individual processes and transformations to which its insight went to meet over the phyletic time $^{7}$ ). The second one focuses on the relationship of dependency between the psycho-

${ }^{6}$ By phylogenesis is meant: the ongoing non-linear (non-predictable, non-reversible, non-reproducible) process of diversification and integration to which undergoes the biological phenomenon from its origin, in relation to all the transitions (bifurcations), and to all the dissipative/anticipatory structures/systems solutions, induced by macroscopic (climatological, geological, planetary) and/or microscopic (biophysical and biochemical) state variations, that have affected it, directly or indirectly, in space and time. In this perspective, phylogeny resembles less to a tree with different branches and more to a basin of attraction of the chaotic type (riddled basin of attraction) with different attractors and different dissipative/anticipatory structures/ systems. [Messori 2011, 2015, 2016]

${ }^{7}$ For a full discussion, see my article: Messori, C. (2016) From Continuity to contiguity. On the Genesis of Consciousness, Culture and Oral Language. Journal of Consciousness Exploration and Research, 7(2), 163228. 
dynamics and the structural and functional properties of the NS in general and of the CNS in particular, i.e. of the human neuro-dynamics.

The three acceptations, psychic-mental-cognitive, of the function intelligence will be discussed separately, first from the point of view of neuro-dynamics (Section 4 and 5) and then from the psycho-dynamics point of view (Section 6).

For convenience, the three definitions of the function referred to in section 6 , will be provided at the end of that section. Advance use of the three adjectives meets these definitions, to which I refer.

Following (Section 7) will be provided a definition of intelligence that, in the absence of an established significant neuro-anatomical correlation, will be formulated within the framework of the interpretative physical model of the mental phenomenon which I have set in [Messori 2012B, 2013, 2015, 2016], that I'm going to summarize in Section 3.

As a general preamble to what I am going to expose, I would point out that from the perspective of this study, the phylogenetic changes, transformations and differentiations, genotypical and phenotypical, structural and functional, are never to be understood as the product of a purposeful design or plan, neither natural nor otherwise intended. On the contrary: 1) they are always the expression of solutions that thanks to an uninterrupted relationship of continuity, in time and space, are intertwined with all the solutions already adopted and with those adoptable (anticipatory systems), even when they are the result of a phylogenetic bifurcation ${ }^{8}$; 2) are always nonlinear and transient solutions (in relation to their evolution over phyletic times); 3) are always stationary but unstable solutions (in relation to the mutability of the unconditioned and conditioned habitat variables); are always intermediate solutions between adaptation and exaptation $^{9}$; 4 ) are always solutions that for their becoming rely on the poietic action

${ }^{8}$ Under the thermodynamic profile, the countless functional and structural solutions that characterize the biological varieties, produced in the course of the phylogenetic diversification in general, and in particular that of the Cambrian, represent as many dissipative structures far from thermodynamic equilibrium. Stationary states to the phase boundary between order and chaos, that if carried further away ( $\rightarrow$ mass extinctions) from thermodynamic equilibrium, may experience a crisis, or bifurcation point, in which the system prefer to move away from the steady state, evolving into some other unpredictable state of stability. [Messori 2015] ${ }^{9}$ The concept of exaptation was introduced by paleontologists Stephen Gould and Elisabeth Vrba in 1982, to indicate the possibility that in nature the relationship between organs and functions is potentially redundant, in order to allow that a tract developed for a certain adaptive reason, can be "co-opted" or converted to a function even completely independent from the previous one. This functional cooptation, which complements and does not replace the gradual action of implementation of natural selection, was named by Charles Darwin "pre-adaptation" and was renamed by Gould and Vrba with the neologism exaptation, means precisely that some innovations, appeared during the course of phylogeny, may not be the result of a process of selection toward that specific function, but the reuse for other purposes of an existing structure. An example is the origin of the wing, which originally worked as a structure for thermoregulation, to then be "recycled" for the flight. Another example is the organs and systems involved in the phonation. Phylogenetically the organs and apparatus involved in phonation are all born for other purposes than vocalization or singing. Over the course of phylogenetic diversification, the organs for the production of breath have been formed to supply lungs with air; the larynx appeared in Mammals as gate between the bellows and the external environment; the vocal channel is the first part of the alimentary canal; the organs for phonetics articulation have as a primary function that of chewing; the nasal resonance cavity correspond to the upper respiratory tract and the soft palate has the main task to prevent the backflow of food. Several examples of functional cooptation have recently been found also at the molecular and cellular level, for example in relation to the role of astrocytes in neurogenesis, which will be taken into account in Section 4 . 
(which produces development and structure) exerted by the self-organization on the genesis and transformation of the forms of manifestation.

Continuity, transience, instability and non-linearity are properties that do not diminish but increase the possibilities of adaptation of biological systems to the continuity, transience, instability and non-linearity of the geo-environmental conditions, on a small and large-scale. Without them, for example, ecosystems would not have had, and would not have, any chance to re-invent and re-adapt themselves to the geo-environmental scenarios produced, e.g., by glaciations and by the various mass extinctions, which have followed in phyletic times as a consequence of natural disasters.

A scenario, the one that emerges from the perspective of investigation adopted here, in contrasts with the supposed quasi-finalistic and quasi-linear consequentiality of the phyletic lineage suggested by the Darwinian and neo-Darwinian arboreal model of phylogeny.

\section{The Physical Dimensions of the Universe}

Each element of reality, at any level of observation, is a micro system of relations, set in a macro system of relations. Nothing of what we observe exists in and by itself. Everything we can think of, observable and unobservable (i.e. virtual), instead of being made up of building blocks, or even quantized, in its ground state consists of tenso-relationship systems.

To clarify the meaning of these statements, and the effects they may have on the interpretation of the issues also examined in this work, we first need to set the ontological scenario within which to place the energy phenomenon, as well as the biological, neurological and mental one.

According to my Endo-Dynamo-Tensive Model [Messori 2012B], the establishment of a physical dimension based on a relational dynamic it arises as a consequence of a cosmogonic event, namely the supraliminal auto-perturbation (tensorial symmetry breaking) of a primary, irreducible and intrinsically dynamic state of tension ${ }^{10}$, ontologically assumed as the physical background from which emerge all the differences in potential, all the interactions or forces and all the physical and psychical relationships. A second supraliminal perturbation (tensorial transition ${ }^{11}$ ) leads to the constitution of the spacetime system of correlative interactions between gradients of tension that we know as relativistic dimension or dimension of an exited tension- gradients distribution or ET-GD (mass and energy free dimension) (Figure 1). A third supraliminal pertur-

\footnotetext{
${ }^{10}$ In its non-exited regime it correspond to a state devoid of structure (continuous, isotropic, homogeneous, imperturbed) and super-symmetrical, of a tension (tension super-symmetry) qualifiable as Implicated Tension (IT), which bears within it a protodynamic principle, whose meaning leads to the Aristotelian concept of "dynamis". In Aristotele by dynamis is meant the potency correlated to the action (energheia), efficient cause connected to the movement and its quantitative and qualitative effects, the inherent potency or intrinsic possibility of a body to be translated in an action (energheia) that may be realized or not, a value of reality only possible with respect to the real action realized. Among the energy dimension IT-dynamics leads to the notion of quanta of action.

${ }^{11}$ In cosmogonic transitions, the state of tension is ensured step by step by symmetry breaking. The centrality of tension in the biophysics of biological systems, which is based on symmetry breaking's rules (e.g. enantioselectivity), is evidenced, for example, by the crucial role of superhelical tension in DNA supercoiling, an essential aspect, not yet well understood, of genomic architecture [18] [19].
} 


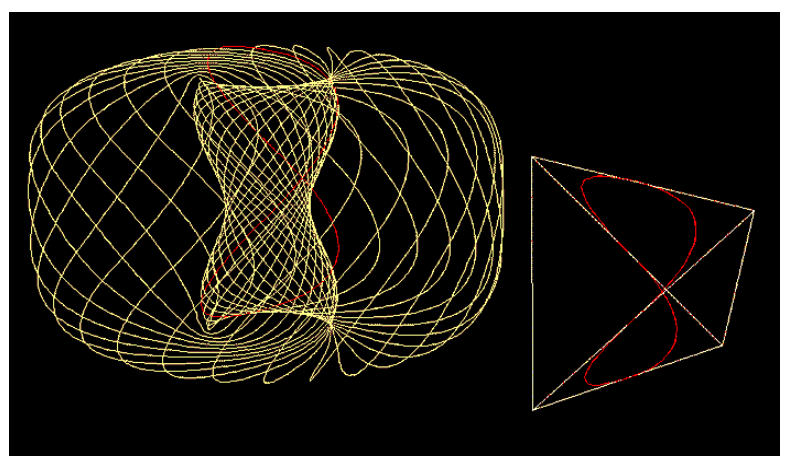

Figure 1. Image source: http://harmoniouspalette.com/TetMold.html. Biaxial or tetra-toroid, also coined as external toroid warped around an internal poloid, as drawn here has 27 identical loops. Compared with ordinary toroid coil, the main differences are twisted loops instead of the plain toroid loops and the involuted "donut hole". While 27 closed loops are presented to show a tetrahedron relationship, all loops can be one continuous twisting line. ET-GD structure corresponds to a non-local (diffuse) holographic cavity resonator with toroidal-poloidal topology consisting of a differentiated (tangent tensions $\rightarrow$ toroid) and undifferentiated (qualia $\rightarrow$ poloid) fractal- tensor foam, wrapped around a wormhole (vortex) lacking in structure and dynamics (emptiness/void/vacuum). ET-GD structure constitutes the basic space-time fabric upon which are grafted all the physical and para-physical phenomena (psychism) that qualify the dimensions of the manifestation. All successive bifurcations are derived from this one via homomorphism (basic dynamicstructural equivalence) and translate the dynamic and structure of the ET-GD, by contextualizing it [Messori 2012B].

bation leads to the constitution of a non-exited energy-quanta gradients distribution (zero point quantum field), from whose perturbation (supra-liminal oscillatory motions and/or charge densities of energy-quanta and impulse) takes shape the quantum and supra-quantum dimension (the dimension of the exited energy quanta-gradients distribution or EEQ-GD), where the relation (ship) is somehow explicated and may possibly be observed and directly experienced.

Every object or phenomenon of the quantum dimension is assimilable to a vibrational system (describable via a mathematical tool known as wave function), that vibrates with a certain frequential configuration, a certain oscillatory or phase modality (rhythm of oscillation) and a certain intensity, maintaining an uninterrupted local and non-local relationship of interference with other vibrational systems.

The phenomena of interference between the oscillatory modalities of the energy flows and impulse, involved in the perturbation/excitation of the quantum field, give rise to coupling-phase (oscillatory resonance ${ }^{12}$ ), able to trigger the phase transitions that lead, according to QED (Quantum Electrodynamic Field Theory), to the struc-

\footnotetext{
${ }^{12}$ In physics resonance or coupling-phase is a condition under which an oscillating system responds to an alternative driving force with the maximum amplitude. Such condition may exist when the frequency of the driving force matches the natural (non-damped) oscillatory frequency of the system. Thus, in case of an imposed oscillating electromagnetic field, a biological system (e.g., a cell) will respond in a measurable manner only to those exogenous oscillations (i.e. alternative driving force) that match the natural (endogenous) EM oscillations of such system.
} 
turing of matter (Coherent Domains vs. Incoherent Domains). In particular, each localized (in space and/or in time) form of confinement (tensorial, energetic, massive, subatomic, atomic, supra-atomic, biological, cosmological), i.e. delimited by a bound$\operatorname{ary}^{13,}$ is a tenso-vibrational micro-environment and corresponds to an oscillator or a resonant cavity (cavity resonator), a stationary system organized around a particular tensorial/frequential configuration of perturbations (tensions/oscillations), existing thanks to the relationships of interference it has with the endogenous and exogenous tenso-vibrational environment ${ }^{14}$.

The final result is the diversification of the structuring of the phenomena affecting the exited energy quanta-gradients distribution (EEQ-GD) regime in four orders of phenomena, relatively autonomous and independent, associated with just as many physical varieties, the first belonging to the territory of the ET-GD, the second to the territory of the EEQ-GD, and the other two to the territory of the Hyper (cosmological) and Middle Dimension (H-MD):

- Tensorial phenomena (tensorial varieties. differentiated vs. undifferentiated tensions; qualia; images);

- Energy phenomena (electrodynamic varieties: wave fields and matter fields; waveparticle duality; anti-symmetrical/chiral composite quantum states and symmetrical/achiral composite quantum states);

- Condensed matter phenomena (thermodynamic varieties, gas/liquid/solid, and chemical varieties, inorganic/organic);

- Biological phenomena (autopoietic varieties).

The condensative varieties of the EEQ-GD and of the H-MD derives from different correlations among tensorial gradients on the one hand, and, on the other hand, from different correlations among anti-symmetricall chiral composite quantum states (fermions ${ }^{15}$ ) and symmetrical/achiral composite quantum states (bosons), corresponding

\footnotetext{
${ }^{13}$ Every structuring process of a system of correlations endowed with a degree of subsistence (condition of resonance) such as to make it distinct and/or distinguishable (even when not observable) from the context of the relationships it forms part of; in general, a confinement process is equivalent to a phenomenon of localization. ${ }^{14}$ In this sense the terrestrial environment is to all effects a tenso-vibrational environment and every biological structure/system corresponds to an oscillator/ resonant cavity tuned on the particular tenso-vibrational configuration of the environment to which it belongs.

${ }^{15}$ The particles described by symmetrical wave functions are known as bosons and obey the statistics of Bose-Einstein. The particles described by anti-symmetrical wave functions are known as fermions and obey the statistics of Fermi-Dirac. Quantum-relativistic mechanics demonstrates that the property of being described by symmetrical or anti-symmetrical wave functions depends on the nature of the particles. In particular it is unequivocally linked to their spin: 1) particles with semi-whole spin are fermions (e.g. electrons, protons, neutrons); all the elementary particles that make up matter are fermions; 2) particles with whole spin are bosons (e.g. photons). All the elementary particles responsible for the forces that hold fermions together are bosons. Since the exchange of two identical particles is mathematically equivalent to the rotation of each particle by $360^{\circ}$, the symmetrical nature of the wave function depends on the spin of the particle after the rotation operator has been applied to it. Particles with whole spin do not change the sign of their wave function after a $360^{\circ}$ rotation, as a result, the wave function sign of the system as a whole does not change. Particles with semi-whole spin do change the sign of their wave function after a 360 rotation. In accordance with the Pauli exclusion principle, two fermions cannot share the same quantum state, while bosons can. This translates into a strong resistance to fermion compression. This resistance creates the rigidity of ordinary atomic matter.
} 
to different coherent oscillatory configurations (domains of oscillatory coherence or Coherent Domains, CD) that oscillate with a non-linear pattern to the rhythm impressed by a carrier frequency modulation.

On quantum and supra-quantum level, the weft of each energy phenomenon, condensed or rarefied, originate from the ongoing and self-organized warp of tensorial gradients constantly regenerated similar to themselves (in order to keep a global quasisymmetrical condition) by the interaction between the quasi-continuous (fractal) and non-uniform distribution of monopolar/achiral gradients of non-hertzian potential (bosons $\rightarrow$ symmetrical wave function ${ }^{16}$; scalar component of the electromagnetic wave) and the quantized and non-uniform distribution of dipolar/chiral gradients of hertzian potential (fermions $\rightarrow$ anti-symmetrical wave function; vectorial component of the electromagnetic wave).

Furthermore, the four orders of phenomena relatively autonomous and independent affecting the EEQ-GD regime, can interact thanks to three types of correlative dynamics or couplings:

- Phase Conjugate Dynamics (of the type Frequency-Phase Correlative Dynamics);

- Spin Coniugate Dynamics (of the type Phase-Tension Correlative Dynamics);

- Tension Coniugate Dynamics (of the type Tension-Tension Correlative Dynamics)

However, the global balanced relationship that sets up this interfacing, namely the fact that the forms taken by energy-matter always remain similar to themselves (self similarity) is endowed by a cascade of unbalanced relationships. That is to say that locally (in space and/or in time) the universe of (self-similar) transformations, where nothing is created and nothing is destroyed, it is constantly shifted towards the component polarized in space or towards the component polarized in time. When this has to happen we speak of transitory quantum or supra-quantum symmetry breaking. Moreover, when this unbalanced state becomes stationary we may observe some very strange phenomenon, e.g. cosmological black holes when it is dramatically shifted towards the figures of interference polarized in space (space collapses), and living systems when it is shifted towards the quasi-tensorial figures of interference polarized in time. That is: in biological systems it prevails the component polarized in time and are themselves polarized in time.

According to this model, then, there aren't pieces of a mosaic (building blocks), but relationship systems (that in the ground state corresponds to pure tenso-relational systems). Their identity and their existence is determined by being part of a particular system of relationships, which manifest themselves solely in the correlative and functional availability of a complex series of energetic and/or tensorial relationships. Talk about

${ }^{16}$ Postulate of symmetrization: The observables of a system of identical $\mathrm{n}$ particles are represented by autoadded operators invariant through any permutation of the particles. The pure states of the system are represented by Hilbert space vectors which are symmetrical (bosons) or anti-symmetrical (fermions) due to the exchange of any pair of particles. The states of a quantum system of $n$ particles can therefore be either symmetrical or anti-symmetrical due to the exchange or permutation of interacting particles. This interaction is also called exchange force (or Heisenberg-Majorana exchange force) and is attractive (plus sign) for symmetrical states and repulsive (minus sign) for anti-symmetrical states. 
hidden codes or pieces of thoughts (e.g., we have neurones that can generate electrical impulses, and these electrical impulses correspond to thoughts) or consciousness, without specifying that it is a scientific artifice, which can be provisionally adopted to facilitate the investigation of a certain object of study, it is a falsification of the mental phenomenon.

\section{From Protoplasm to Nervous System: Part One}

Living systems are transient systems tuned on state variations or stimuli (perturbative events as frequency variations, phase variations, tension variations) of the internal and external environment: their subsistence depends on their ability to adapt to (specific) applied perturbations. To adapt they must be, at first, selectively excitable. Selectivity is an essential condition for the existence of an autopoietic system. An example of biological selectivity is enantioselectivity. Biological systems are enantioselective, i.e. they strictly select the enantiomeric forms of the molecular species of which they consist (biological reactions synthesize and use always and only one of the two enantiomeric forms of a given molecule).

The enantioselectivity of biological systems is the reason for their homochirality, namely the presence of groups of molecules that have all the same enantiomeric configuration (e.g., the amino acids are all in the levorotatory configuration, while the ribose and deoxyribose of the nucleic acids have only dextrorotatory configuration).

Enantioselectivity, homochirality and autopoiesis of biological phenomenon seem to be related to the phase transition of water from the ordinary coherence of its liquid state to the semi-crystalline or glassy and super-coherent state of biological water [20][28], the liquid medium within which the protoplasm (the colourless, translucent, viscoid, gelatinous and semi-fluid matrix of all cells) is formed.

The water $\left(\mathrm{H}_{2} \mathrm{O}\right)$ is the third most common molecule in the Universe (following the $\mathrm{H} 2$ and $\mathrm{CO}$ molecule), and its chemical structure, based on the hydrogen bond, it is studied long since. Many models have been proposed but none has yet been able to explain a number of water properties considered anomalous [29].

The Quantum Field Theory (QFT) and QED (Quantum Electrodynamic Field Theory) have produced a vision of water in a liquid state as a medium, which for a peculiarity of its molecular electronic spectrum reveals itself as an essential tool for long range communications, being able to change its supramolecular organization in function of the interaction with the environment.

The electromagnetic fields that are trapped within the Coherent Domains (CDs) of water and within their coherent matrices [30], produce electromagnetic potentials that regulate the phase of the entire system, which in turn gives rise to selective attractions between the molecules of the solute.

"......the $C D$ of liquid water", writes Emilio Del Giudice ${ }^{17}$ [translation from Italian is mine], "unlike the CDs of other molecular species, is susceptible to give rise to a large

${ }^{17}$ Del Giudice, E., Tedeschi, A., (2009) La respirazione dell'acqua come base della dinamica della vita. Atti del XXIV Congresso di Omeopatia, Omotossicologia e Medicina Biologica, Milano, 16 Maggio-Roma, 23 Maggio -Sessione di Ricerca Scientifica sull'Acqua. 
number of excited states. Arises, consequently, the possibility of a further level of coherence, generated by the collective oscillation of a plurality of CDs of water, between two own configurations: a coherence between CDs, namely Super-coherence, that on one hand makes it grow the size of the related region by the tenth of a micron of the elementary CDs of water, up to microns of the cells, to centimeters of the organs, or to the meters of higher organisms. How it happens the collective oscillation of these CDs? Given the plurality of the excited levels of the CD, it is able to withdraw from the environmental noise small amounts of energy, transforming them into coherent vortices of quasi-free electrons. The duration of these vortical excitations can be very long (days, weeks, months), since because of the coherence the internal friction is zero, there are no collisions and the CD cannot dissipate energy in thermal form. Given the long duration of these excitations, it is possible to accumulate a large number of them within the domain, such as being able to accumulate a capital of 1 million euro in coins from 1 cent. Each vortex is a motion of electrons [31] [32], that is, electrically charged particles, which gives rise to the appearance of a magnetic moment, which in turn aligns with the environmental magnetic field, which in the final analysis can also be the Earth's magnetic field. The vortices therefore cannot cancel each one another, but add up coherently, then transforming low-quality environmental energy (high entropy), in high quality coherent energy (low entropy), capable of inducing, as predicted by Szent - Gyorgyi, the electronic excitation of the molecules surrounding the CD. When the energy accumulated in the $C D$ reaches the level corresponding to the energy of chemical activation of biomolecules present in the CD in the water, it develops, for electromagnetic resonance, the energy transfer [33] [34] [35]. The CD will discharge, thus completing its oscillation, and a set of well-defined chemical reactions take place, ruled by an electromagnetic resonance law, and not by the diffusive regime based on the random motions and collisions of molecules typical of non-biological chemistry. This makes it possible to achieve two objectives simultaneously.

-it allows the oscillation of the water CDs, a necessary precondition for the onset of the Super-coherence, extending the correlation radius to biologically relevant distances;

-it creates a biochemistry not governed by random collisions between molecules, but by a code of mutual recognition and recall among molecules based on long-distance electromagnetic interaction.

The biochemical reactions become therefore selective, that is obedient to specific organic codes, and fast. Furthermore, the chemical energy released during chemical reactions once taken from the CD modifies its characteristic frequency of oscillation, causing in turn the change of the type of molecular species able to resonate with the domain. We have, therefore, a non-stationary coherent regime, but that evolves over time, generating in turn a time-dependent biochemical framework. This way, we get a qualitative agreement with the main manifestations of the living dynamic, which appear incomprehensible in the conceptual framework of a chemistry ruled by diffusive regime and by the accidental collisions. The overall dynamics of the living appears to 
emerge, ultimately, by the oscillatory rhythm of water and by the related energetic events".

All biological envelopes, from cell membrane to epithelial tissue, contain this aqueous phase in a semi-crystalline state (likened to the liquid ice) or are perfused by it. It is water in a particular phase of quantum organization (oscillatory super-coherence) that gives it a high capacity to:

1) retain electronic charges, in the form of vortical excitations of quasi-free electrons, storable as energetic reserve;

2) induce an electronic and protonic long-range and long life excitation of the different molecular species available, enabling their selective activation and mutual attraction ( $\rightarrow$ enantioselective absorption);

3) convert mechanical vibrations (phonons) in quantum of electromagnetic energy (photons) and vice versa (piezoelectric effect).

According to this line of research, the resonance phenomena induced by the electromagnetic field occupy a central role in the explanation of the establishment of the super-coherent phase of biological water, which in living organisms results also in plasma membranes, amniotic fluid $(\mathrm{AF})^{18}$, cerebrospinal fluid (CSF), interfacial and intracellular water (the viscous, glassy layers of interfacial water contiguous to biological surfaces are called Exclusion Zones, EZs, in which the solutes are not able to penetrate) (Figure 2), the latter being considered a key characteristic that has been exploited by Nature in setting up a mechanism able to match the quite different time scales of protein and solvent dynamics [36], and a key characteristic for intercellular signal transmission [37] [38] [39] [40].

\subsection{From Protoplasm to Nervous System: Part Two}

One of the general properties of the protoplasm, the biological material of which all cells are constituted (chemically composed by water, electrolytes, proteins, lipids and carbohydrates), possibly originated by the structuring process to which has undergone biological water [34] [41] [42] [43] during the early Archean period of the Precambrian Era, is irritability, namely the ability to sense an external stimulus and react to it. An organism consisting of a single cell (like the amoeba) reacts to a contact by contracting its cytoplasm. Irritability, however, is not an answer, but a cell must be irritable to respond to a stimulus. In vertebrates, for example, epithelial cells, when stimulated, can respond by secreting, muscle cells by contracting, and nerve cells (neurons) producing

\footnotetext{
${ }^{18}$ In human placental mammal the intrauterine development process, first embryonal and then fetal, takes place inside a sac ( $\rightarrow$ placenta development) filled with AF. AF increases progressively in relation to embryonic/fetal growth, and until the 10th week of gestation is formed essentially by maternal plasmatic ultra-filtrate. From the 10th to the 20th gestational week, it has a composition very similar to that of the fetal plasma, which diffuses into the amniotic fluid through the thin and nonkeratinized embryonic/fetal skin. From the third quarter is largely formed by the urine and by cutaneous and lung transudation of the fetus. In the past it was thought that the AF was limited to performing the function of cushion and settling tank of embryonic and fetal metabolic degradation, but recent studies have revealed that plays an immune action and occupies a leading role, together with the CSF, in the embryo development of the NT, i.e. neurogenesis, and of the CNS, morphogenesis.
} 


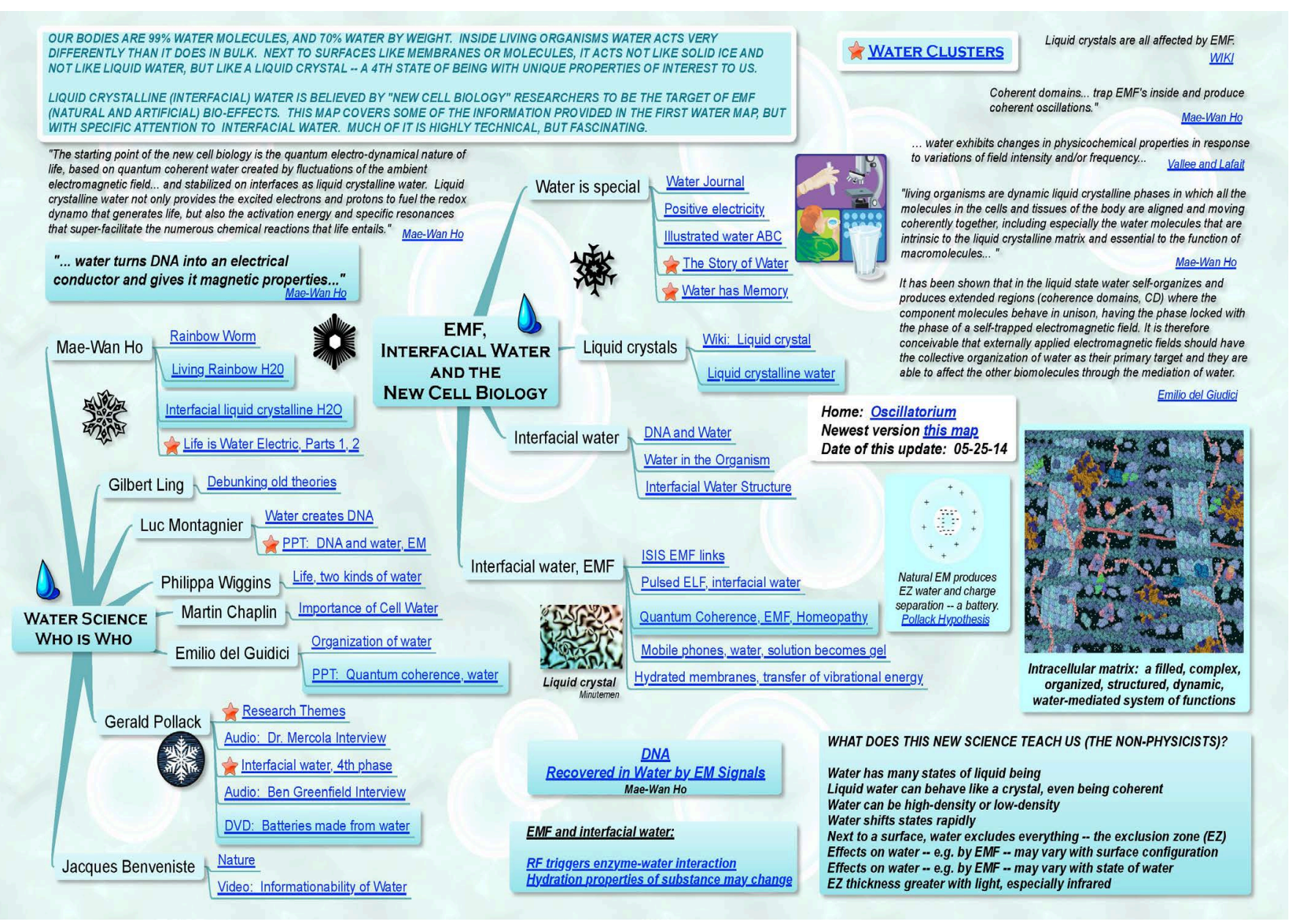

Figure 2. EMF, Interfacial water and the new cell biology. Image source: www.oscillatorium.com/id54.html.

and/or conducting neuro-electro-chemical impulses, or action potentials ${ }^{19}$, that propagate along their extensions (axons and dendrites) at variable speed. In multicellular organisms of the animal kingdom (from Metazoa down to us), in which the various functions are distributed among different and increasingly specialized tissues, this characteristic property of irritability is acquired and perfected to the maximum degree by neurons that, together with other cells, such as glial cells [44], form the Nervous Tissue (NT), whose morphological, anatomical and functional diversification and integration leads to the establishment of the Nervous System (NS) [45] [46].

In animals with a structural and functional neurological degree of integration perfectly efficient and relatively diversified, such as Coelenterates, for example jellyfish and polyps, the NS is limited to a network of neuromuscular elements, equipped at the same

\footnotetext{
${ }^{19} \mathrm{~A}$ neuro-electro-chemical impulse or nerve impulse or action potential is a soliton wave that propagates along the cell membrane (thanks to a chain process of transmembrane modification of permeability which coincides with transient variations of the membrane ionic concentration), with constant amplitude and intensity but a variable frequency and speeds (the speed of propagation is directly proportional to the diameter and the degree of myelination of the nerve fiber; the frequency is directly proportional to the excitatory state of the neuron).
} 
time with sensitive and contractile capacity. In animals with a higher structural and functional degree of diversification and integration, as in some Shellfish, the NS is organized into more complex and selective structures, constituted by differentiated cells (that is, which have acquired particular structural characteristics fulfilling the function to be performed) grouped in depth, under the integuments, to form a chain of ganglia (neuronal clusters) at the side of the digestive tract. With the appearance of vertebrates the subdivision of the NS in central organs (notochord $\rightarrow$ neural tube) and peripheral structures (nerves and receptor organs), prefigured in some species of marine animals at the border between invertebrates and vertebrates (such as Pikaia gracilens, considered the fossil ancestor of the Amphioxus), undergoes a decisive stabilization and the central units of it (encephalus and spinal cord) are contained within a osteo-fibrous case represented by the skull and the vertebral canal.

Characteristic of the animals with bilateral symmetry (such as the species Homo) is a body structural plan arranged along a longitudinal axis, with the right half which is roughly the mirror image (but not overlapping) of the left half. An animal with bilateral symmetry can move more effectively than an animal with radial symmetry (such as Coelenterates) that, in turn, is better adapted to a sedentary life. Another characteristic of animals with bilateral symmetry is having a spatial orientation of the axial type, i.e. arranged longitudinally between two ends, a front (cephalic or rostral) and a posterior (cauda). Having a forward end is a characteristic of very active animals. In such organisms many of the sensory cells (nervous cells specialized in receptorial-stimulus specific functions) are concentrated in the front end of the body with a greater concentration of nervous cells specialized in functions other than those stimulus specific: thanks to this particular neuro-anatomical organization the organism is found to be practically oriented in a preferred direction, forward, on the basis of which the animal organizes its motor behavior.

\subsection{Nervous System and Functional Role of Neurons and Glial Cells}

The amazing thing about the brain is that it permits, for example, the sensory-motor co-ordination of the whole interaction, the hormonal regulation which ensures the maintenance of corporeal integrity, and so on, but the notion of neuronal correlates of mind as such is, to use the words of Alfred Norton Whitehead, "an inopportune concretization". Francisco Varela

In the non-linear process of phylogenetic diversification, nerve cells play the role of receptor units of zoological individual of tissue organisation, whose dual selective function on state variations (stimuli) and functional interface between innervated tissues, supports and integrates the function exerted by the catalytic cellular core (CCC), formed by the Golgi apparatus, the centrosome (MTOC, Microtubule Organizing Center [47]) and microtubules (the structural units of the cell cytoskeleton, polymerized protein highly polarized), on energy-transfer. Cell differentiation which leads to the identification of the specialized cell neuron, responds to a phylogenetic request $(\rightarrow b i$ - 
furcation), which at a certain stage of evolution (Cambrian), gave a new degree of freedom to the biological systems in their relationship with the environment: the zoo-logical line of neuro-dependent behavioural relationship.

Diversification of behavioral strategies require then cell differentiation, the process by which cells specialize, acquiring or enhancing their ability to perform a specific function $^{20}$. With cell differentiation the whole clusters of unicellular organisms that have colonized the planet until then, distributed over one or more areas and linked by a common adaptive and bio-energetic gain, found the way to come together (a process of in-formation governed by the self-organization of specific associative patterns induced, in the short and long range, by resonance phenomena involving various kind of cellular and molecular species) to become localized cells colonies, specialized and joined by a common structural and functional link, defined and identified in the construction of different tissues, organs and systems of organs, that operate coherently and in synchrony for the survival and unity of the multicellular system. The biological unit (cell), from being a composed, integrated system delimiting (membrane) a multitude of subcellular structures and molecular units, became an integrated specialized cellular ecosystem (multi-cellular organism), consisting of differentiated cells, specialized according to the role and function they must play to be part of a choral unit.

In vertebrates, CNS functional and morphological organization follows exactly this pattern. At increasingly specialized and diversified behavioral strategies, pointing at species-specific CNS functions, also corresponds a distribution of different, morphologically and/or functionally specialized neuron-glial colonies, whole groups of cells belonging to species-specific Coherent Domains of the CNS basin of attraction ${ }^{21}$, each of them in a relation of continuity with all the others, where the single behaviour is prescribed by the colony's choral interference network, and described by a unanimous and synchronised dynamic, based on self-organized biophysical processes occurring via Phase Conjugate Dynamics, Spin Coniugate Dynamics, Tension Coniugate Dynamics.

Throughout the NT of invertebrates and vertebrates, neurons are invariably found in close association with glial cells (or glia-like cells in primitive animals, with very simple cellular organization), a type of cells that have been co-opted (exaptation), at least in vertebrates, to perform functions other than the original ones. Indeed, until recent

\footnotetext{
${ }^{20} \mathrm{All}$ cells have the fundamental properties of living protoplasm, i.e. metabolic activity (synthesis, respiration), excitability, the conduction of excitation, the ability to receive stimuli from the environment and to respond by movement, secretion or other ways, plus the capacity to reproduce. When a cell specializes it loses none of these fundamental properties related to biological life, but it differs because it potentiates one of these properties. Generally speaking, when a cell specializes in a particular function, it also modifies its morphology.

${ }^{21}$ Roughly speaking, an attractor of a dynamical system is a subset of the state space to which orbits originating from typical initial conditions tend as time increases. It is very common for dynamical systems to have more than one attractor. For each such attractor, its basin of attraction is the set of initial conditions leading to long-time behavior that approaches that attractor. Thus the qualitative behavior of the long-time motion of a given system can be fundamentally different depending on which basin of attraction the initial condition lies in (e.g., attractors can correspond to periodic, quasiperiodic or chaotic behaviors of different types). Regarding a basin of attraction as a region in the state space, it has been found that the basic topological structure of such regions can vary greatly from system to system. [Credit: Ott, E. (2006) Scholarpedia. http://www.scholarpedia.org/article/Basin_of_attraction]
} 
times it was believed that only neurons and synapses were to carry out the functional activity of the NS and CNS, but increasing evidence accumulated in the last decades suggest that this role should be shared with glial cells, the number of which is significantly higher than that of neurons (ratio of about 10-50:1), which regulate the neuronal synaptic responses associated with learning and memory processes and share with neurons the role of mediators in the genesis of brain functional skills [48] [49] and occupy a prominent place in the neurogenesis of NT and in the evolution (e.g. via astroglyal lineage cells [50]) and architecture of the $\mathrm{CNS}^{22}$. The term glia includes non- neuronal cells known to play several functions in the maintenance of neural homeo- stasis in both central (CNS) and peripheral (PNS) nervous system. The main glial cell types are, in the CNS, astrocytes, oligodendrocytes and microglia and, in the PNS, Schwann cells, enteric glial cells and satellite cells. Evolutionary origins of glia are possibly linked to organizational needs, including structural support, by-product removal, phagocytic functions, developmental programming and circuit modulation [51]. Glia probably evolved from neuro-ectodermal cells, possibly from a mobile lineage or independently in different regions of the body, having multiple functions in both developing and mature nervous system [52] [53]. A notable exception is microglia, which are part of the immune system and enter the CNS from blood circulation early in an organisms development. Radial glia is generated before neurogenesis (see below), the process of generating functional neurons from neural and glial precursors ( $\rightarrow$ stem cells), very active in the pre-natal period ( $\rightarrow$ development of the CNS) and relatively active also in post-natal period ( $\rightarrow$ neuronal repair processes) [54], and guide neuronal migration in the developing brain. In mammals, radial glial cells disappear or become astrocytes within days to weeks after birth, but in non-mammals they persist into adult- hood as radial glia [55] [56].

Neurogenesis is a complicated process of generating functional neurons, which involves, in addition to neural and glial precursors, mature glial cells, the CSF and ependymal cells ${ }^{23}$. During embryonic development, the vast majority of neurons and a large number of glial cells are generated in the germinal zones or germinal matrices or Neu-

\footnotetext{
${ }^{22}$ The CNS appears at the beginning of the 3rd gestational week as stretched ectodermal thickening, the neural plate, located in the dorsal-central region of the embryo, in front of Hensen's node and the primitive streak. The neural plate is lifted, forming two reliefs, the neural crests. These crests are then joined incorporating part of the AF in which the embryo is immersed, giving shape to a tubular structure, the neural tube. The amniotic fluid incorporated in the neural tube is then turned into CSF, initially by the neuroepithelial cells (NECs) and by the radial glial cells (RGCs), and subsequently by the choroid plexuses (CHPs), folded structures residing in the brain ventricles that consist of a single layer of highly active epithelium sandwiching an elaborate vascular network (this vascular-neural composite controls the passage of molecules into the CSF, which impacts neurogenic zones throughout life), which start to develop when the embryo reaches the $8 \mathrm{~mm}$ (9th week of gestation). The neural plate and later the neural tube, continue to elongate caudally until it is sketched the entire embryo axis. At the end of this period, 29th day, the CNS, of tubular structure, consists of a cephalic enlarged end, the brain, and a long caudal portion, the spinal cord. At the 6th week of gestation the cephalic end has three expansions, the primary encephalic vesicles: the forebrain or front brain; the midbrain or medium brain; and the hindbrain or rear brain.

${ }^{23}$ Specialized epithelial cells, which create a selectively permeable barrier (ependyma) between the CSF and nervous tissue, and that, according to recent studies, can generate neuro-stem cells (NSCs), pluripotent cells that can differentiate and become neurons or glial cells.
} 
rogenic Niche [57] [58] [59], the micro-anatomical environment that depending on the period of neuronal development (prenatal $\rightarrow$ postnatal) may contain endothelial cells, ependymal cells, stem cells, astrocytes, microglia, neurons and mature descendants of adult neural precursors, and that functionally controls their development in vivo. In the early phases of cytogenesis, the germinal zones are located primarily at the ventricles surface (ventricular zone, VZ). While embryonic development proceeds, on the surface of $\mathrm{VZ}$ forms a germinal subventricular zone $(\mathrm{SVZ})^{24}$, which together form the ventricu-lar-subventricular zones (V-SVZs) [60] [61] [62].

As the cerebral hemispheres enlarge, and the distance to be travelled by the cells increases, most of the neurons that will form the cerebral cortex migrate to their destinations along specialized radial glial fibers (RGFs) that span the entire thickness of the hemisphere from the ventricular surface to the pia $^{25}$.

After birth neurogenesis is carried out by the specialized activity of the neurogerminative subgranular zone (SGZ) of the dentate gyrus of the hippocampus [59] [63] [64].

\section{The Autopoietic Psycho-Neuro Dynamics}

I view the brain not as a box with compartments that contain sadness, joy, color, texture, and all the other

"objects" and categories that one might think of.

Instead, I envisage it as a constantly shifting dynamic system; more like the flow of a river in which patterns emerge and disappear, than a static landscape.

This is an entirely different image from the brain as a computer with stored contents or subroutines to be called up by a program.

In nature's pattern-forming systems, contents aren't contained anywhere but are revealed only by the dynamics.

Form and content are thus inextricably connected and can't ever be separated.

J.A. Scott Kelso

Through NT and NS, the organism may develop the ability to interface biunivocally the territory of tension with the territory of energy, so as to give way to new relational and adaptive strategies (new compared to the non- or pre-neurological relational and adaptive strategies). In this sense, NT-NS as a unit is a selective interface between the

\footnotetext{
${ }^{24}$ The cells that will form the deep cerebellar nuclei and the Purkinje cells of the cerebellar cortex are generated in germinal zones in the subventricular region of the fourth ventricle. The cells that form the deep cerebral nuclei are formed along the ventricular surface of the diencephalon (the future third ventricle) and from the ganglionic eminence of the telencephalon (the future site of the caudate nucleus). The diencephalic germinal zone will produce cells that form the thalamus, hypothalamus, and globus pallidus, whereas the cells produced in the ganglionic eminence will form the striatum (caudate nucleus and putamen), amygdaloid complex, and claustrum.

${ }^{25}$ The orderly production and migration of the cells, from the germinal zone to the cerebral cortex along the RGFs, led to the concept of neuronal-glial vertical units. This unit includes the germinal zone which produces the cells destined for a certain region of the cortex, the cells themselves, and the bundles of radial glial fibers which guide the cells to their final destination.
} 
two territories, energy $\leftrightarrow$ tensorial, and the original function assigned by cell differentiation to nerve cells is receptorial and meta-physical (of interfacing between one plan and the other of reality): that is, NT and the NS, regardless of the number of anatomical/functional units (neurons, glial and the like) involved, are condensed (fermionic) systems specialized in interfacing the Exited Tension-Gradients Distribution (ET-GD) with the Exited Energy Quanta-Gradients Distribution (EEQ-GD), and viceversa, for adaptive purposes. Photons (massless objects) and phonons (quantized mode of vibration) exchanges generated by the neuro-electro-chemical activity are at the basis of this dynamic of interfacing.

With respect to the territory of energy, NT-NS makes a selection (receptor function) species-specific upon the state variations or stimuli (perturbative events, e.g. frequency variations, phase variations, tension variations) from the external and internal environment, making the organism appropriately sensitive to a certain range of state variations and not to others. Moreover, NT-NS is specialized in transforming, upon kinematic similarity, the chemical and physical state variations of the environment, in spatial and temporal neuro-electro-magnetic phase correlations. With respect to the territory of tension NT-NS it transmutes the stimuli from frequencial pattern (receptor activity $\leftrightarrow$ neu-roelectro-chemical activity) in isomorphic pattern of spin, on which operates a specific $\mathrm{Ho}$ lographic Strange Attractor ${ }^{26}$ [12] [13] [14], which transmutes the pattern of spin in tensorial pattern, and viceversa, and sets the functional correlations between them, valid under the biological and/or neurological and/or psychic standpoint.

The mind(body) system, i.e. the psycho-neuro dynamics characterizing an alive and vibrating human being (all neurologic organisms exhibit proto-mindedness, but not all of them are minded [65]), it can be seen as an autopoietic system strung between the Exited Tension-Gradients Distribution (ET-GD) and the Exited Energy Quanta-Gradients Distribution (EEQ-GD), governed by the syntropic action of an Holographic Strange Attractor, able to develop different degrees of integration and to carry out different functions, that responds to the laws of psychodynamics of non-equilibrium, that constantly redefines itself and that internally it sustains and reproduces itself. The autopoietic dynamic of mind (body) system is organized around biophysical autocatalytic patterns (i.e. self-accelerated) regulated by continuous and non-linear fluctuations of selective tension transfer between intra and extra environment. The trans-neurological selectivity is the central element of the autopoietic psycho-neuro dynamics. There is a diffuse and integrate neurological intercellular relationship, supported by a dense network of physical and chemical signals (via synapses, gap junctions, intra- extracellular fluid, plasma membranes, CSF [66] [67]) capable of interacting with the environmental substrate so as to produce the net of soliton waves, i.e. neuro-electro-chemical impulses or nerve impulses or action potentials, that qualifies the sensory/ receptor system activity. Selective tension transfer between intra and extra environment is associated with the production and

\footnotetext{
${ }^{26} \mathrm{~A}$ spatiotemporal center of tensorial stability, dimensioned as a fractal and configured as a hologram, around which it occurs the indeterministic variability of the coupling relationships (frequency-phase coupling on quantum level; phase-tension coupling on quantum-relativistic level; tension-tension coupling on relativistic level).
} 
transduction of tensorial signals (coherent scans of tension state variations or tensostimuli) that come into play in the tensorial interconnection and in the biophysical processes of Nervous System tuning (via Phase Conjugate Dynamics, Spin Coniugate Dynamics, Tension Coniugate Dynamics): that is, all psyco-neuro information dynamic is carried, transformed and made available as holographic spin-wave interference patterns on the surfaces of higher order hyperspace fields (e.g. on a hypersphere, a structure embedded in a $4 \mathrm{D}$ space, equipped with a double donut-like shape, which is invisible in the usual 3D dimensions [68] [69] [70] [71]), which are non-locally resonant with the intermediate EM field generated by the CNS activity (Figure 3 ).

In general, a Nervous System defines and separates this network of interactions from the environment so that it can realize an autonomous but not isolated unit, namely a living neurologic organism. Humans, and to some extent also other vertebrates, are engaged in engramming a sensorineural warp of tensions on which it can weave the weft of a variably integrated state of mind.
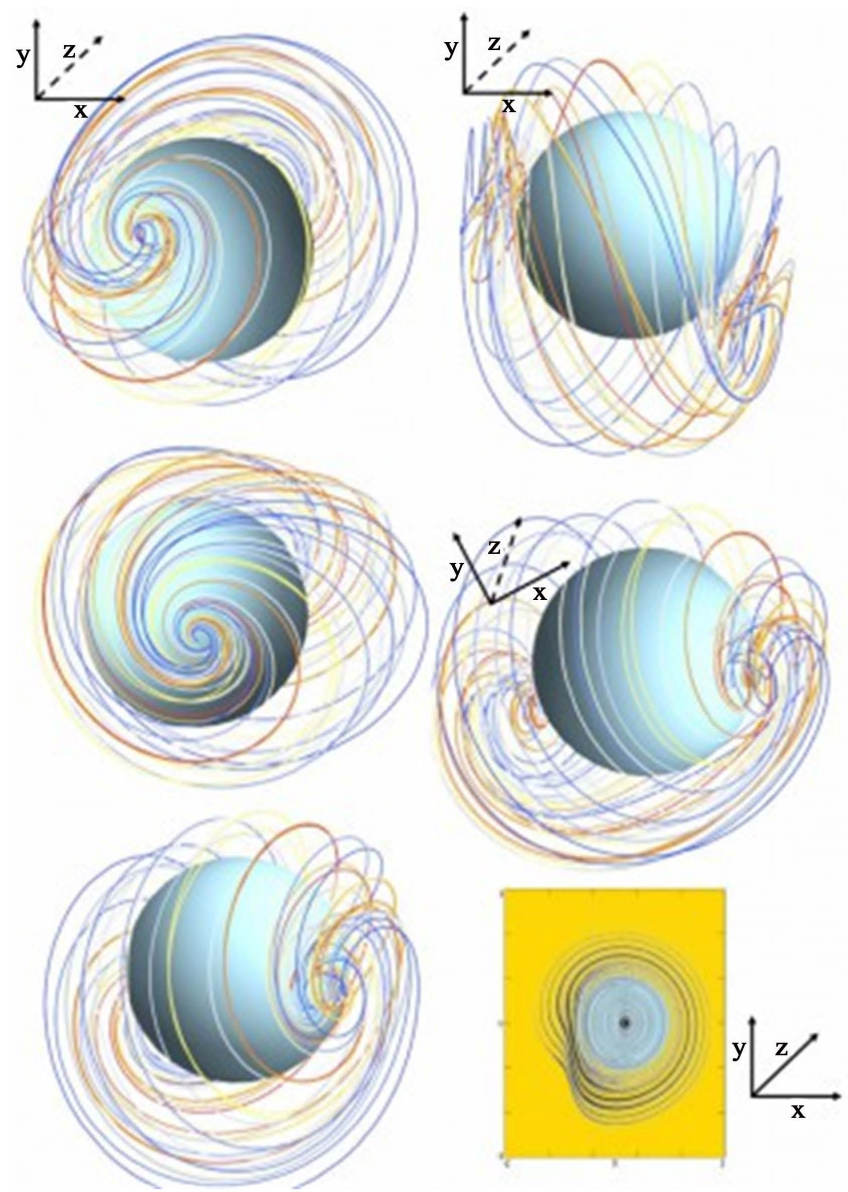

Figure 3. Image source:

http://arturotozzi.webnode.it/products/strange-attractors-and-tori-embedded-in-a-brain-phase-s pace/. STRANGE ATTRACTORS AND TORI EMBEDDED IN A BRAIN PHASE SPACE. The brain is a system at the edge of chaos equipped with nonlinear dynamics and functional energetic landscapes. 
In vertebrates, the sensorineural response common to all neurological organisms is processed and integrated, e.g. via super-complex anticipatory systems ${ }^{27}$, to become perception (perception being a function more integrated than sensitivity which is a function more integrated than reacting which is a function more integrated than excitability), which in humans is associated to sensing, the mental substrate on which thinking is grafted (see next Section).

\section{Psycho-Dynamics of the Psychic-Mental-Cognitive Functions}

Like a river whose eddies, vortices, and turbulent structures do not exist independent of the flow itself, so it is with the brain.

Mental things, symbols and the like,

do not sit outside the brain as programmable entities, but are created by the never ceasing dynamical activity of the brain. The mistake made by many cognitive scientists is to view symbolic contents as static, timeless entities that are independent of their origins. Symbols, like the vortices of the river, may be stable structures or patterns that persist for a long time, but they are not timeless and unchanging.

\section{J.A. Scott Kelso}

From the Lower Paleolithic to the first half of the Middle Paleolithic, the action produced by the encounter between human intelligence and the environment was limited to ensure the performance of adaptive phylogenetically prescribed functions, with few but significant deviations from the phylogenetic prescription, which already from the first half of the Lower Paleolithic attest to how the human species were phylogenetically endowed with the possibility to overcome the behavioral stereotypy, to which are irreducibly bound all other animals [Messori, 2016]. Throughout this period, about two million years, the psychic basin of attraction around which it has revolved the human psychodynamics, was essentially composed by what C.G. Jung calls sensing-intuition bipolar psychic dimension ${ }^{28}$, an irrational psychic dimension composed by the psychic

\footnotetext{
${ }^{27}$ Rosen, R. (2012) Anticipatory Sistems. Philosophical, Mathematical and Methodological Foundations. Second edition (first edition 1985), Springer.

${ }^{28}$ Sensing is one of the four poles of mental functions introduced by C.G. Jung in his Psychological Types, where he introduces a hierarchy of mental functions in two mental bipolar dimensions (dichotomies). These are sensing (attentiveness by means of the sense organs) coupled to intuition (awareness in unconscious way or being aware of unconscious contents) and thinking (function of intellectual cognition; the forming of logical conclusions) coupled to feeling (function of subjective estimation). When one of the poles of the two dichotomies predominates over the rest of the poles, it defines the dominant function of a person or a community of persons. The sensing-intuition dichotomy represents the way in which an individual receives information. According to Jung sensing and intuition are called irrational functions. Sensing refers to the means by which an individual knows something exists, derived by his/her neuropsychological-sensory system. Sensing, i.e. attentiveness by means of the sense organs, is the species-specific mental function common to all vertebrate, upon which it develops their relationship with the environment and each other. Intuition is knowing about something without conscious understanding of where that knowledge comes from. The irrational function, according to Jung, is typical for mental and perceptual activity that predominantly (and, for the most part, unconsciously) operates with opportunities, i.e. various possible outcomes and sensations result from some premises and sensations, mostly driven by unconscious processes. In humans, all the forms of behaviour, including cognitive behaviour, and communication, including oral language, are rooted on the psycho-physical plane of (tension) sensing-intuition [Messori 2016].
} 
function sensing, that due to the action carried out by the six sense organs ${ }^{29}$ allows the individual to maintain an adequate state of attention to, and behave depending on, the environmental stimuli (energy and tensorial state variations), and the psychic function intuition, which it provides the individual a pool of raw psychic contents, prescriptive but not descriptive (archetypes ${ }^{30}$ ) that lend themselves to nourish, and to be fertilized by, the relationship with the environment. With the psychological birth of human individuality (Middle Paleolithic) [Messori 2016] and the subsequent acquisition of a distinct identity from the environment (relationship of contiguity), indispensable prerequisite for the establishment of what we call self-consciousness ${ }^{31}$, the psychic environment changes radically its structure and the bipolar psychic dimension sensing $\leftrightarrow$ intuition is integrated with another fundamental bipolar psychic dimension, in this case rational, composed by the psychic $\rightarrow$ mental function thinking, that through recourse to logical processes tend to derive mind-facts from the raw object (archetype), and the psychic $\rightarrow$ mental function feeling, which tends to assign a value to things depending on the characterial predispositions and expectations of individuals who experience $\mathrm{it}^{32}$.

From this time onwards the human being begins to entertain a dialogical relationship with the environment, mediated by the state of tension that develops between the phylogenetic content ( $\rightarrow$ independent variable), which gives-form (in-form) to the bipolar psychic dimension sensing $\leftrightarrow$ intuition and the epigenetic content ( $\rightarrow$ dependent variable), which in-form the bipolar psychic dimension thinking $\leftrightarrow$ feeling, and to identify itself as an agent of mediation between Earth and Sky, between the consistency of a reality perceived through the six sense organs, and supported by the psychic function sensing $(\rightarrow$ Earth), and the consistency of a reality intuited through archetypal psychic

${ }^{29}$ The brain is the organ of sense for tensorial state variations, or tensorial stimuli [Messori, 2012B, 2015, 2016].

${ }^{30}$ Archetypal structures underlie all recurrent, "typical" (panhumanly typical, not culturally or personally typical) ideas, images, categories, situations, and events that arise in experience. They contain no inherent content, but exist "at first only as forms without content, representing merely the possibility of a certain type of perception and action". Archetypes may manifest as "a priori, inborn forms of 'intuition"”. And as the instincts impel us to act in a distinctly human way, so do the archetypes impel us to perceive and understand the events we instinctively respond to in a distinctly human way. (Laughlin, C.D. (1968) Archetypes, Neurognosis And The Quantum Sea. Journal of Scientific Exploration, 10, 375-400.

http://jung2.org/ArticleLibrary/Archetypes,\%20N\%20and\%20Quantum\%20Sea.pdf)

${ }^{31}$ The essential requirement for making culture is given by recognize oneself as individuality distinct from the environment, a psychological birth that has come to maturity only during the Middle Paleolithic, after a long psycho-relational and psycho-biological process of individuation, already started 1.7 to 1.2 million years ago with Homo Abilis and almost entirely spent on the fulfillment of the requirements associated to survival and caring for offspring. After this long process of individuation was perhaps starting from the later generations of Homo heidelbergensis (archaic humans) and certainly with Homo Sapiens (and therefore from about 300-150 thousand YA onwards) that the psychic territory of the imaginific, from phylogenetically differentiated as it was it turned out more and more epigenetically differentiated, by the sedimentation of a psychic complex relatively autonomous and independent, which we call epigenetic function of the real (pre-rational and pre-verbal) or human self-consciousness [Messori 2012A, 2016].

${ }^{32}$ The thinking-feeling dichotomy refers to how an individual processes the information. The function thinking allows a person to understand the meanings of things. The function feeling is the method by which a person qualifies the value of conscious activity. This process relies on logic and careful mental activity. According to Jung thinking and feeling are called rational function, because it typical for mental activity that consciously operates with judges or analyzes received information. 
contents, and supported by the psychic function intuition $(\rightarrow \mathrm{Sky})^{33}$.

Animated by the dynamics of the new psychic $\rightarrow$ mental environment, the exercise of intelligence comes out from the incubation stage where has operated for about two million years, entering a phase in which the epigenetic learning process $(\rightarrow$ dependent variable) integrates and shapes the phylogenetic prescriptive action ( $\rightarrow$ independent variable), bringing to maturity the formation of mental functions and adaptive and supra-adaptive skills that can also make use of relational modules other than the one (phylogenetically prescribed) that has characterized the social life of human communities from Lower Paleolithic to Middle Paleolithic, i.e. different from the imitative module, based on the imaginific function ${ }^{34}(\rightarrow$ bipolar psychic dimension sensing $\leftrightarrow$ intuition) and mediated by the human acoustic-musical faculty ${ }^{35}$ [Messori 2012A].

Between Middle and Upper Paleolithic, the action carried out by the imitative module in the relational life of human communities of Homo Sapiens et Faber was built, initially, by the symbolic module and then (from Upper Paleolithic onwards) by the ideative module, based on the namesake functions, now yes, cognitive.

Based on these premises, the psychodynamic distinction that can be made between psychic function, mental function, cognitive function, is the following:

\footnotetext{
${ }^{33}$ When the human's state of mind, endowed by the tenso-relational dynamics ruled by sensing-intuition, which give rise to the specific anatomical and functional organization of the human's neuropsy-chologicalsensory system, is integrated by the thinking-feeling mental bipolar dimension, the phylogenetic scenario change dramatically, as the human-animal and his/her environment from being an interconnected/ interacting/interfering unit becomes subject-object of a relationship conditioned by an internal represen- tation ot the external reality conceived, initially via symbolling (from the second half of Lower Paleolithic) and then also via semantics abilities (from late Middle Paleolithic onwards), in the settling process of the psychological complex relatively autonomous and independent that we call epigenetic function of the real or self-consciousness.

${ }^{34}$ From the Greek eidolopoios or idolopeo, which produces images. The term imaginific refers to self-organization of physical massless and energyless objects on the tensorial plane, i.e. spectra of coherent correlations of spin-internal motion, which we call images. Mental images are spectra of coherent correlations of spin-internal motion, derived from spectra of coherent correlations of spin-magnetic moment/angular momentum, which in turn are derived from the ongoing spectra of coherent phase correlations, produced by the neuroelectro-chemical activity, generated in the relationship between environment $\leftrightarrow$ PNS $\leftrightarrow$ CNS $\leftrightarrow$ environment.

${ }^{35}$ All biological systems, from the less to the most integrated, are organized, functionally and structurally, for adaptive purposes, in order to respond, as a fundamental priority, to the needs for interferential efficacy. Such needs may have to deal with the geo-environmental and bio-environmental variables, which determines the biological limits and possibilities, conveyed by the genic setting, to generate interference, that is transfer of tension-energy from a biological oscillating system, endowed with its peculiar oscillation frequency and rhythm, to another. One of the ways for this explication to take place, is on the acoustic plane, which in vertebrates results in an acoustic-musical interference attitude and in the structures to support it. All anatomical and functional systems responsible for issuing detailed sound scans endowed with coherence (signals) are vibrational environments themselves, configured as cavity resonators. The circumstantiated emission of complex and coherent sounds, characterized by their own rhythm and timbre (signal), by an animal considered in its natural habitat it establishes, via sound-consonances and dissonances, an interferential adaptive relationship with other animals and with the acoustic qualities of the vibrational environment of belonging. The human animal can rely on a particular adaptive and supra-adaptive aptitude, essentially intuitive and imitative, pre-symbolic, pre-rational and pre-verbal, which I call human acoustic-musical faculty, in reproducing the rhythmical matrices that enliven the environment in which he/she lives, a peculiar and ready to use, innate (unconscious) faculty that it takes place on the human psychic plane of sensing-intuition, that makes the human being a polyphonic and polyrhythmic animal.
} 
-The psychic function is the relational capacity, distinctive, involved in the phylogenetically prescribed arrangement of the human psychic environment (insight), i.e. its species-specific tensorial basin (the particular tensorial warp on which it can be weaved the energy weft characterizing the genus Homo) (Figure 4) in whom are dissolved all the phase transitions (bifurcations) who went to meet the psychism ${ }^{36}$ (C.G. Jung) of the biological phenomenon in the course of phylogeny [Messori 2000, 2012A, $2015,2016]$, passing through the neurological transition up to the bifurcation that led to the emergence of the genus Homo.

-The mental function is the explicated, modal and procedural, form of the psychic function. It refers to the process of information that went to meet the human psychic $\rightarrow$ mental environment in the relationship with the environment.

De facto, the psychic function and the mental function have always been compresent (what varies is the prevalence of one over the other), and they point to the Jungian concept of archetype (psychic raw material) and to that of collective unconscious.

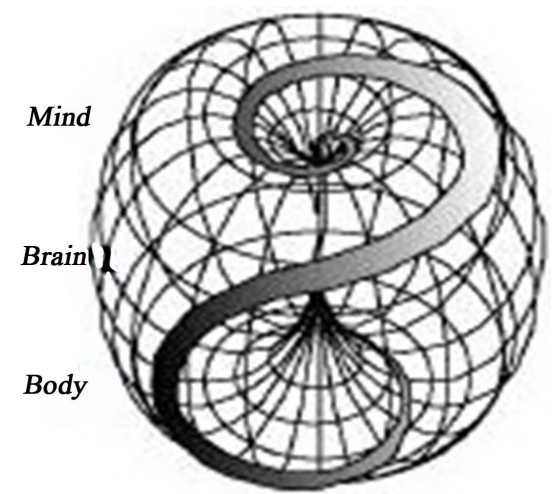

Figure 4. The human tensorial basin (of attraction), i.e. the mind-brain-body (de-localized) system, reproduces the fundamental structure characterizing the EEQ-GD, which in turn reproduces the fundamental structure of the ET-GD. The fundamental structure of the EEQ-GD is a unitary fractal and resonant object, a toroidal-like cavity resonator, consisting of an outer frequency weft (Phase Conjugate Dynamics $\rightarrow$ Body), an inner spin weft (Spin Coniugate Dynam$i c s \rightarrow$ Brain) and a tensorial nucleus (Mind $\rightarrow$ Tension Coniugate Dynamics). On the ET- GD level, the precursor of this fractal object, is a diffuse toroidal vortex consisting of spin-inter- nal motion (zitterbewegung, self-dynamism not dependent upon external factors), a stationary and fractional (non-quantized) entity within which the motion impulse is only latent. Subjected to a torque and rotational action (induced by reflection symmetry breaking) this diffuse toroidal vortex transform into a bi-polar quantum-relativistic object, named Twisted-Pinched Hysteresis Loop (T-PHL) [Messori 2012B], in which the spin-internal motion is de-symmetrized in spin-up and spin-down. The final outcome (EEQ-GD) is a continuous and non-uniform cyclotronic motion of dextrorotatory vs. levorotatory spin-angular momentum, that in passing through the de-localized point of self-intersection of the loop (the eight-like strip highlighted in the picture) decussate and reverses sign, moving seamlessly from negative values to positive values. The mirror symmetry breaking leading to the biological T-PHL is at the basis of the enantioselective absorption mediated by biological water, which determines biological homochirality.

\footnotetext{
${ }^{36}$ The term psychism refers to the warp of fractalized, stationary and unstable patterns of undifferentiated tensorial fractions, on which engages a network of differentiated tensorial fractions, named tangent tensions, by combination of which flows the spacetime fabric (exited tension-gradients distribution, ET-GD).
} 
From the psycho-dynamic point of view, these two functions qualify the constitutive arrangement, and the dynamics, of the psychic $\rightarrow$ mental environment, which precedes the psychological birth of the genus Homo (Middle Paleolithic).

-The cognitive function, is the relational capacity, gained from the particular differentiation, and subsequent arrangement, of the psychic $\rightarrow$ mental environment, after the psychological birth and, in particular, by the development of oral language and by the maturation of cognitive thinking ( $\rightarrow$ bipolar psychic dimension thinking-feeling $\rightarrow$ logical-abstract thinking), understood as the ability to program significant actions with respect to the ability to formulate meaningful purposes, by resorting to the exercise of ideation and ratiocinative discrimination.

\section{Intelligence}

In those places, the nicknames, the jungle names, were defined by psychologists as "construction of a double", the purpose of which was to dissociate children from guilt and totally detach them from their self prior to recruitment; in Sierra Leone some childish units called themselves "cyborgs" denoting consciously themselves as semi-human machines, devoid of feelings.

Alberto Sanza

The phylogenetic history of the genus Homo, of which we can claim to know, in its fossil record, at least five species of which only one, Homo Sapiens, currently living, began about 2.5 million years ago with Homo Abilis. To date, the study of brain evolution in the fossils it has been restricted to the analysis of the variations occurred over the phyletic time 1) by the cranial capacity, 2) by the surface layers of the cerebral volumes, such as the marks left on the intracranial wall by the cerebral convolutions and by the vascular structures (arteries and veins) that have been shaping the surface of the cerebral cortex, and 3) by the bony structures that serve as docking or anatomic reference for the soft tissues, all variables that are suitable to be used in studies of comparative paleo-neuro-anatomy, but that does not give any information on the deep areas of the brain, and especially do not allow to trace the phylogenetic evolution of the psychic functions. In recent times, the transition to digital morphology and multivariate analysis techniques, interpreted according to the principles of morphological integration, has increased dramatically the possibility of anatomical reconstruction, allowing the study of intracranial form (endocast ${ }^{37}$ ) to go well beyond the simple analysis of brain volume. Many encephalic variations can therefore be interpreted as non-neural consequences of encephalization process (increased cranial capacity in relation to body size), or as structural consequences of changes associated with the facial structures or with those at the base of the skull. Other changes are more directly interpretable as real changes in

\footnotetext{
${ }^{37} \mathrm{An}$ endocast is a casting for which the cranial cavity serves as a mold. In mammals, endocasts look remarkably brainlike, resembling a fresh brain with the dura mater in place.
} 
cortical areas, with possible functional relationships of cognitive character. Specifically, the morphological changes in the higher areas of the brain (frontal and parietal lobes) are typically more sensitive to real neural changes. Changes in the conformation of the frontal and parietal lobes are described in the most encephalized human species, i.e. in Neanderthal populations and in anatomically modern humans. In the latter, the changes of the parietal volumes are extremely noticeable, and are also associated with marked changes in cerebral vasculature. In return, the lower brain structures (temporal, occipital and cerebellar lobes) are subjected to biomechanical stresses of the base of the skull, which often determine changes in its shape, without necessarily affect neural functional changes. In any case, there are not elements to establish a deterministic and linear correspondence of cause and effect between the diversification of brain functions and the morphological and structural changes which went to meet the CNS in vertebrates. Moreover, there aren't elements to establish a relationship of proportionality between the encephalization index and the quality or quantity of brain functions expressed by an organism, just as there is no proportional relationship between the number of its genes and the quality or quantity of its behavioral strategies ${ }^{38}$.

Furthermore, since the relationship between mind and brain, i.e. between psyco-dynamics and neurodynamics, it has nothing to do with transmission of information, exchange of meaningful messages, decryption of encrypted code, computational encoding of bit of information and the like (if the genesis of the biological and mental phenomena had to be based on the precepts of Information Theory, there would be no living nor minded systems at all), the definition of intelligence that follows is formulated according to the psycho-dynamics line of investigation.

The word intelligence comes from the Latin intellegentia (or intelligentia), which in turn comes from the verb intellëgo (o intelligo), derived from lëgo with the addition of the prefix, which is also a preposition, inter, "in the middle", "between" (or of the adverb intus, "inside"). The Latin verb lëgo is related to the Greek verb légo with which it shares the same Indo-European root ${ }^{*} L A G-/{ }^{*} L E G$-, which it expresses the idea of harvest, collect, gather, pull together and tune different things, connect, from which the Sanskrit lagati, "to bind to", "to attach". From this root, the Greek légo has developed the lexical form of the "collecting-braiding-talk" (meaning "to collect, braiding the sounds and tune the words in a phrase"), that refers to the dichotomous nature of the lógos, the ordering function of the lógos as "logic-word" (speech, truth, principle, law, reasoning, discernment), and the function as medium of the logos "magic-word" (intelligible sound, en-chant, mythos, mantra, symbol), while the Latin lego has developed the lexical form of the "collecting-braiding-read" (meaning "collecting with the eyes the graphic signs of the words, pronouncing them within onself, in silence"), from which lex, "the law" (as code that collects and concretises the general principles of the ius), and re-ligio, "religion" (renew the bond with alterity).

From this etymological reconstruction it turns out that, in summary, intelligence indicates the ability to select "between and inside", weaving and tuning to each other and

${ }^{38}$ The Amoeba dubia (a unicellular organism) has a genome consisting of 670 billion nucleotide bases, more than 200 times the size of the human genome. 


\section{within them different things.}

Keeping this definition and developing it so as to obtain a sufficiently articulated and unequivocal definition, we can say that: with the term intelligence we refer to the capacity, congenital and acquired, conscious and unconscious, of an individual or a community of individuals, to select, tune, polarize and apply his/her/their psychic $\rightarrow$ mental $\rightarrow$ cognitive functions, in the identification and/or in carrying out the behavioral and/or knowledge strategies, adaptive or supra-adaptive, utilitarian or non-utilitarian, as a response, genetic or epigenetic, to the relational and/or knowledge needs that arise by the encounter (made of dependent and independent, predictable and unpredictable variables) between the pressure exerted by the inner environment (insight) and the pressure exerted by the external environment.

As such, intelligence does not indicate the abilities for this or that specific relational skills, or competence or psychic-mental-cognitive function, neither it refers to the degree of development of one of them, nor can be broken down into a more or less extended series of capacities. It makes no sense, rather it is patently incorrect, to speak of logical-mathematical intelligence, verbal i., spatial i., musical i., kinesthesic i., emotional i., etc. Intelligence is a global, choral, systemic, property not sectorizable nor factorizable, which reveals itself in the encounter between the action taken by the individual/ community into the environment and on the environment, and the action exerted by the environment on the individual/community and in the individual/community, a global property that depends on the possibilities, capacities and tendencies of those who exercises it, in front of all the variables involved.

Is it possible to relate or even identify intelligence with the computing and storage capacities of an intelligent machine? What can it have in common with the artificial, computational, predictable, reversible, reproducible, planable and controllable skills' simulation carried on by the ASSS?

\section{Conclusions}

Western man has no need of more superiority over nature, whether outside or inside. He has both in almost devilish perfection.

What he lacks is conscious recognition of his inferiority to nature around him and within him. He must learn that he may not do exactly as he wills. If he does not learn this, his own nature will destroy him. He does not know that his own soul is rebelling against him in a suicidal way C.G. Jung

The epigenetic learning makes use of behavioral strategies and tools of investigation and knowledge that since the Bronze Age are placed and proceed on two different but complementary logic levels, one rational and the other irrational [1] [72]. One is dynamic, facing outward, oriented to the elaboration and adoption of strategies and tools in knowledge of the physical, material, formal, sensible, immanent reality, circumscribed by the dimension of rationality and meanings. When intelligence and learning 
are developed on this plan, the skills that are acquired are supported predominantly by the thinking-feeling bipolar psychic dimension, and meet practical, utilitarian, government-like aims. The other is static, facing inward, oriented to the elaboration and adoption of strategies and tools in knowledge of the immaterial, informal, meta-physical, magical reality, circumscribed by the dimension of irrationality and symbols (the excess of meaning, the alterity). When intelligence and learning are developed on this plan, the skills that are acquired are supported predominantly by the sensing-intuition bipolar psychic dimension, and can lead to two different solutions: one makes use of strategies and exterior tools of investigation mediated by the senses, but it is devoid of practical and utilitarian purposes (e.g. the artistic production), while the other is totally detached from both practical and utilitarian purposes and from strategies and external tools of investigation mediated by the senses (ecstatic experiences, contemplative states, deep meditative states).

All civilizations born out of metallurgy revolution had to, have to and will have to deal with these two logic levels, from whose elaboration takes shape their respective internal representation of external reality, their culture, social and cultic organization.

That is not by chance that in the last years of his life, Auguste Comte (1798-1857), the ideologist of positivism, writes the positivist Catechism and founded the Positivist Church, where are transposed the doctrinal, ethical and liturgical elements of the Catholic Tradition. In fact, by doing so, he closes the circle around his vision, positivist, of the metaphysic applied for practical purposes (Messori 2015), the same vision that animates contemporary scientist and technicians, who want to emulate the theologians that almost two thousand years ago managed to bundle the alterity in the consubstantiality of the Christological perichoresis, i.e., they made immortal what is mortal and mortal what is immortal, an alchemical operation remained unequaled, at least until today, the Age of the Super-Enlightenment, in which science seeks to give life and consciousness to the machines!

Who knows, maybe to realize how silly it is to describe biological minded systems adopting the criteria applied by techno-science to build and program the so-called intelligent artificial systems, i.e. idiot machines, claiming that we should learn from their intelligence; meaning that we have to be idiots as they are, and we'll have to wait for a child to be accompanied by parents at the opening party of the 2040 AI Olympic Games, and while the crowd cheers all around, the puzzled child asks to the mother: Mom, but do they know that those over there are just machines?

\section{References}

[1] Messori, C. (2016) From Continuity to Contiguity. On the Genesis of Consciousness, Culture and Oral Language-Part I of IV. Journal of Consciousness Exploration \& Research, 7, 163-228.

https://www.researchgate.net/publication/295857703_From_Continuity_to_Contiguity_On _the_genesis_of_consciousness_culture_and_oral_language_Part_I_of_IV

[2] Messori, C. (2000) Il Sole e la Luna. Sulla Natura dei Simboli e della Mente Umana. Federico Ceratti Editore, Milan. 
[3] Messori, C. (2012) Dalla Facoltà Acustico-Musicale alle Origini del Linguaggio Orale Fino al Predominio della Cavità Orale che Genera il Mondo sulla Cavità Uterina che Genera la Vita. Il Minotauro, Persiani Editore, Bologna, 2, 6-43.

[4] Mencattini, A., et al. (2014) Speech Emotion Recognition Using Amplitude Modulation Parameters and a Combined Feature Selection Procedure. Knowledge Based Systems, 63, 68-81. http://dx.doi.org/10.1016/j.knosys.2014.03.019

https://www.researchgate.net/publication/261328157_Speech_emotion_recognition_using amplitude_modulation_parameters_and_a_combined_feature_selection_procedure

[5] Fleischer, J.G. and Edelman, G.M. (2009) Brain-Based Devices: An Embodied Approach to Linking Nervous System Structure and Function to Behaviour. IEEE Robotics \& Automation Magazine, 16, 33-41. http://dx.doi.org/10.1109/MRA.2009.933621 http://www.nsi.edu/ fleischer/fleischer_edelman_ram.pdf

[6] Agbinya, J.I. (2011) Principles of Inductive Near Field Communications for Internet of Things. River Publishers.

[7] Regalado, A. (2014) Military Funds Brain-Computer Interfaces to Control Feelings. MIT Technology Review, May 29.

https://www.technologyreview.com/s/527561/military-funds-brain-computer-interfaces-tocontrol-feelings/

[8] Strickland, E. (2014) DARPA Project Starts Building Human Memory Prosthetics. IEEE Spectrum, August 27.

http://spectrum.ieee.org/biomedical/bionics/darpa-project-starts-building-human-memory -prosthetics

[9] DARPA (2015) Neurotechnology Provides Near-Natural Sense of Touch. DARPA News and Events. www.darpa.mil/news-events/2015-09-11

[10] Sanchez, J. (2015) Restoring Active Memory (RAM). DARPA Program Information. www.darpa.mil//program/restoring-active-memory

[11] Sanchez, J. (2015) Systems-Based Neurotechnoloy for Emerging Therapie (SUBNETS). DARPA Program Information.

[12] Messori, C. (2011) Cells, Neurons, and Qualia: The Holographic Strange Attractor Model. Journal of Consciousness Exploration \& Research, 2, 1417-1437.

https://www.researchgate.net/publication/255696668_Cells_Neurons_and_Qualia_The_Hol ographic_Strange_Attractor_Model

[13] Messori, C. (2012) A Cosmogonic Model of Human Consciousness. Journal of Consciousness Exploration \& Research, 3, 1149-1208.

https://www.researchgate.net/publication/255696726_A_Cosmogonic_Model_of_Human_ Consciousness

[14] Messori, C. (2013) L'Antropologia della Coscienza incontra la Fisica QuantisticoRelativistica: Modello Cosmogonico Endodynamotensivo (EDT) e Mnemopoiesi (MOPS). Volume 1, Il Minotauro, Persiani Editore, Bologna, 1, 7-82.

[15] Messori, C. (2015) Quale Modello per le Neuroscienze-Part. I of III. Volume 1, Il Minotauro, Persiani Editore, Bologna, 1, 25-45.

[16] Messori, C. (2015) Quale Modello per le Neuroscienze-Part II of III. Volume 2, Il Minotauro, Persiani Editore, Bologna, 2, 80-117.

[17] Messori, C. (2016) Quale Modello per le Neuroscienze-Part III. Volume 2, Il Minotauro, Persiani Editore, Bologna.

[18] La Marr, W.A. (1998) The Effect of Supercoiling on Small Molecule-DNA Interactions. Massachusetts Institute of Technology, 1-103. 
https://dspace.mit.edu/bitstream/handle/1721.1/50414/41163964-MIT.pdf?sequence=2

[19] Vologodskii, A.V., et al. (1979) Fluctuations in Superhelical DNA. Nucleic Acids Research, 6, 967-982. http://dx.doi.org/10.1093/nar/6.3.967 http://www.ncbi.nlm.nih.gov/pmc/articles/PMC327745/pdf/nar00444-0159.pdf

[20] Zhou, E.H., et al. (2009) Universal Behavior of the Osmotically Compressed Cell and Its Analogy to the Colloidal Glass Transition. Proceedings of the National Academy of Sciences of the United States of America, 106, 10632-10637. http://dx.doi.org/10.1073/pnas.0901462106

[21] De Ninno, A., Del Giudice, E., Gamberale, L. and Congiu Castellano, A. (2014) The Structure of Liquid Water Emerging from the Vibrational Spectroscopy: Interpretation with QED Theory. Water Journal, 6, 13-25. http://www.waterjournal.org/uploads/vol6/deninno/WATER.2013.13.DeNinno.pdf

[22] Ho, M.W. (2014) Large Supramolecular Water Caught on Camera: A Review. Water Journal, 6, 1-12. http://www.waterjournal.org/uploads/vol6/ho/WATER.2013.12.Ho.pdf

[23] Clegg, J.S. (1982) Alternative Views on the Role of Water in Cell Function. In: Franks, F. and Mathias, S.F., Eds., Biophysics of Water, John Wiley and Sons, New York, 365-383.

[24] Antonenko, Y.N., Pohl, P. and Rosenfeld, E. (1996) Visualisation of the Reaction Layer in the Immediate Membrane Vicinity. Archives of Biochemistry and Biophysics, 333, 225-232. https://www.jku.at/biophysics/content/e54633/e54639/e54665/

[25] Mollenhauer, H.H. and Morré, D.J. (1978) Structural Compartmentation of the Cytosol: Zones of Exclusion, Zones of Adhesion, Cytoskeletal and Intercisternal Elements. In: Roodyn, D.B., Ed., Subcellular Biochemistry, Vol. 5, Plenum Press, New York, 327-362.

[26] Barry, P.H. and Diamond, J.M. (1984) Effects of Unstirred Layers on Membrane Phenomena. Physiological Reviews, 64, 763-872.

[27] Pollack, G.H. and Clegg, J. (2008) Unexpected Linkage between Unstirred Layers, Exclusion Zones, and Water. In: Pollack, G.H. and Chin, W.C., Eds., Phase Transitions in Cell Biology, Springer Science \& Business Media, Berlin, Germany, 143-152.

[28] Tedeschi, A. (2010) Is the Living Dynamics Able to Change the Properties of Water? International Journal of Design \& Nature Ecodynamics, 5, 60-67. www.witpress.com/Secure/ejournals//papers/D\&NE050108f.pdf

[29] Keutsch, F.N. and Saykally, R.J. (2001) Water Clusters: Untangling the Mysteries of the Liquid, One Molecule at a Time. Proceedings of the National Academy of Sciences of the United States of America, 98, 10533-10540. http://www.pnas.org/content/98/19/10533.full.pdf

[30] Murugan, N.J., Karbowski, L.M. and Persinger, M.A. (2014) Serial pH Increments ( 20 to 40 Milliseconds) in Water during Exposures to Weak, Physiologically Patterned Magnetic Fields: Implications for Consciousness. Water Journal, 6, 45-60. http://www.waterjournal.org/volume-6/persinger-summary-2

[31] Meyl, K. (2012) DNA and Cell Resonance: Magnetic Waves Enable Cell Communication. DNA and Cell Biology, 31, 422-426. http://dx.doi.org/10.1089/dna.2011.1415 http://www.k-meyl.de/go/Primaerliteratur/Magnetic_Waves-Enable-Cell_Communication. pdf

[32] Meyl, K. (2012) About Vortex Physics and Vortex Losses. Journal of Vortex Science and Technology, 1, Article ID: 235563.

http://omicsonline.com/open-access/about-vortex-physics-and-vortex-losses-2090-8369.10 00101.pdf?aid=15110

[33] Del Giudice, E. and Tedeschi, A. (2009) Water and the Autocatalysis in Living Matter. 
Electromagnetic Biology and Medicine, 28, 46-54.

http://dx.doi.org/10.1080/15368370802708728

http://www.ncbi.nlm.nih.gov/pubmed/19337894

[34] Voeikov, V.L. and Del Giudice, E. (2009) Water Respiration-The Basis of the Living State.

Water Journal, 1, 52-75.

www.waterjournal.org/uploads/vol1/voeikov/WATER-Vol1-Voeikov.pdf

[35] Brizhik, L.S., Del Giudice, E., Tedeschi, A. and Voeikov, V.L. (2011) The Role of Water in the Information Exchange between the Components of an Ecosystem. Ecological Modelling, 222, 2869-2877. http://dx.doi.org/10.1016/j.ecolmodel.2011.05.017 https://www.researchgate.net/publication/229327908_The_role_of_water_in_the_informati on_exchange_between_the_components_of_an_ecosystem

[36] Pagnotta, S. and Bruni, F. (2007) The Glassy State of Water: A “Stop and Go" Device for Biological Processes. In: Pollack, G.H., et al., Eds., Water and the Cell, Springer Verlag, Heidelberg, German, 93-112.

[37] Agnati, L.F., et al. (1995) Intercellular Communication in the Brain: Wiring Versus Volume Transmission. Neuroscience, 69, 711-726. http://dx.doi.org/10.1016/0306-4522(95)00308-6

[38] Agnati, L.F. and Fuxe, K. (2014) Extracellular-Vesicle Type of Volume Transmission and Tunnelling-Nanotube Type of Wiring Transmission Add a New Dimension to Brain Neuro-Glial Networks. Philosophical Transactions of the Royal Society B, 369, Article ID: 20130505.

[39] Agnati, L.F., et al. (2010) Understanding Wiring and Volume Transmission. Brain Research Reviews, 64, 137-159. http://dx.doi.org/10.1016/j.brainresrev.2010.03.003

[40] Agnati, L.F., et al. (2014) Information Handling by the Brain: Proposal of a New "Paradigm" Involving the Roamer Type of Volume Transmission and the Tunneling Nanotube Type of Wiring Transmission. Journal of Neural Transmission (Vienna), 121, 1431-1449. http://dx.doi.org/10.1007/s00702-014-1240-0

[41] Del Giudice, E. and Vitiello, G. (2006) The Role of the Electromagnetic Field in the Formation of Domains in the Process of Symmetry Breaking Phase Transition. Physical Review A, 74, Article ID: 22105. https://arxiv.org/pdf/cond-mat/0607594.pdf http://dx.doi.org/10.1103/PhysRevA.74.022105

[42] Del Giudice, E., Spinetti, P.R. and Tedeschi, A. (2010) Water Dynamics at the Root of Metamorphosis in Living Organisms. Water Journal, 2, 566-586.

http://www.mdpi.com/2073-4441/2/3/566 http://dx.doi.org/10.3390/w2030566

[43] Montagnier, L., et al. (2015) Transduction of DNA Information through Water and Electromagnetic Waves. Electromagnetic Biology and Medicine, 34, 106-112. https://arxiv.org/pdf/1501.01620v1.pdf http://dx.doi.org/10.3109/15368378.2015.1036072

[44] Jessen, K.R. (2004) Cells in Focus, Glial Cells. The International Journal of Biochemistry \& Cell Biology, 36, 1861-1867.

http://www.ucl.ac.uk/cdb/research/jessenmirsky/publications/IntJBiochem.pdf

[45] Roth, G. and Dicke, U. (2013) Evolution of Nervous Systems and Brains. In: Galizia, C.G. and Lledo, P.M. (eds.) Neurosciences-From Molecule to Behavior: A University Textbook. Chapter 2, Springer-Verlag Berlin Heidelberg, 19-45. http://dx.doi.org/10.1007/978-3-642-10769-6_2

[46] Bucher, D. and Anderson, P.A.V. (2015) Evolution of the First Nervous Systems-What Can We Surmise? Journal of Experimental Biology, 218, 501-503. 
http://jeb.biologists.org/content/218/4/501

http://dx.doi.org/10.1242/jeb.111799

[47] Efimov, A., et al. (2007) Asymmetric CLASP-Dependent Nucleation of Noncentrosomal Microtubules at the Trans-Golgi Network. Developmental Cell, 12, 917-930.

http://www.cell.com/developmental-cell/abstract/S1534-5807(07)00149-9

http://dx.doi.org/10.1016/j.devcel.2007.04.002

[48] Oberheim, N.A., Goldman, S.A. and Nedergaard, M. (2012) Heterogeneity of Astrocytic Form and Function. Methods in Molecular Biology, 814, 23-45.

http://dx.doi.org/10.1007/978-1-61779-452-0_3

[49] Oberheim, N.A., et al. (2009) Uniquely Hominid Features of Adult Human Astrocytes. The Journal of Neuroscience, 29, 3276-3287.

http://dx.doi.org/10.1523/JNEUROSCI.4707-08.2009

www.cns.nyu.edu/events/spf/SPF_papers/Oberheim_et_al_2009.pdf

[50] Robertson, J.M. (2013) Astrocytes and the Evolution of the Human Brain. Medical Hypotheses, 82, 236-239.

https://www.researchgate.net/publication/259586299_Astrocytes_and_the_evolution_of_th e_human_brain

[51] Bondan, E.F., et al. (2015) Glial Cells of the Central Nervous System of Bothrops jararaca (Reptilia, Ofidae): An Ultrastructural Study. Pesquisa Veterinária Brasileira, 35, 685-690. http://www.scielo.br/pdf/pvb/v35n7/1678-5150-pvb-35-07-00685.pdf http://dx.doi.org/10.1590/S0100-736X2015000700014

[52] Allen, N.J. and Barres, B.A. (2009) Glia-More than Just Brain Glue. Nature, 457, 675-677. http://dx.doi.org/10.1038/457675a

[53] Hartline, D.K. (2011) The Evolutionary Origins of Glia. Glia, 59, 1215-1236. http://dx.doi.org/10.1002/glia.21149

[54] Barkovich, A.J., Gressens, P. and Evrard, P. (1992) Formation, Maturation, and Disorders of Brain Neocortex. American Journal of Neuroradiology, 13, 423-446. http://www.ajnr.org/content/13/2/423.full.pdf

[55] Lazzari, M. and Franceschini, V. (2004) Glial Fibrillary Acidic Protein and Vimentin Immunoreactivity of Astroglial Cells in the Central Nervous System of the African Lungfish, Protopterus annectens (Dipnoi: Lepidosirenidae). Journal of Morphology, 262, 741-749. https://www.researchgate.net/publication/8229778_Glial_fibrillary_acidic_protein_and_vi mentin_immunoreactivity_of_astroglial_cells_in_the_central_nervous_system_of_the_Afri can_lungfishProtopterus_annectens_Dipnoi_Lepidosirenidae http://dx.doi.org/10.1002/jmor.10274

[56] Weissman, T., et al. (2003) Neurogenic Radial Glial Cells in Reptile, Rodent and Human: from Mitosis to Migration. Cerebral Cortex, 13, 550-559.

http://cercor.oxfordjournals.org/content/13/6/550.full http://dx.doi.org/10.1093/cercor/13.6.550

[57] Bjornsson, C.S., et al. (2015) It Takes a Village: Constructing the Neurogenic Niche. Development Cell, 32, 435-446. http://dx.doi.org/10.1016/j.devcel.2015.01.010 http://www.cell.com/developmental-cell/pdf/S1534-5807(15)00033-7.pdf

[58] Ma, D.K., et al. (2008) Neurogenic Niches in the Adult Mammalian Brain. In: Gage, F.H., Kempermann, G. and Song, H., Eds., Adult Neurogenesis, Chapter 11, Cold Spring Harbor, New York, 207-225.

[59] Ming, G. and Song, H. (2011) Adult Neurogenesis in the Mammalian Brain: Significant Answers and Significant Questions. Neuron, 70, 687-702. 
www.cell.com/neuron/pdf/S0896-6273(11)00348-5.pdf

http://dx.doi.org/10.1016/j.neuron.2011.05.001

[60] Morshead, C.M., et al. (1994) Neural Stem Cells in the Adult Mammalian Forebrain: A Relatively Quiescent Subpopulation of Subependymal Cells. Neuron, 13, 1071-1082. http://dx.doi.org/10.1016/0896-6273(94)90046-9

[61] Reynolds, B.A. and Weiss, S. (1996) Clonal and Population Analyses Demonstrate That an EGF-Responsive Mammalian Embryonic CNS Precursor Is a Stem Cell. Developmental Biology, 175, 1-13. www.sciencedirect.com/science/article/pii/S0012160696900901 http://dx.doi.org/10.1006/dbio.1996.0090

[62] Doetsch, F., Garcia-Verdugo, J.M. and Alvarez-Buylla, A. (1999) Regeneration of a Germinal Layer in the Adult Mammalian Brain. Proceedings of the National Academy of Sciences of the United States of America, 97, 11619-11624. http://dx.doi.org/10.1073/pnas.96.20.11619

[63] Oberheim N.A., et al. (2006) Astrocytic Complexity Distinguishes the Human Brain. Trends in Neurosciences, 29, 547-553. http://dx.doi.org/10.1016/j.tins.2006.08.004

[64] Fiorelli, R., et al. (2015) Adding a Spatial Dimension to Postnatal Ventricular-Subventricular Zone Neurogenesis. Development (Cambridge, England), 142, 2109-2120.

http://dev.biologists.org/content/142/12/2109 http://dx.doi.org/10.1242/dev.119966

[65] Maiese, M. (2015) Embodied Selves and Divided Minds-International Perspectives I Philosophy \& Psychiatry. Oxford University Press, Oxford.

[66] Vigh, B. and Vigh-Teichmann, I. (1998) Actual Problems of the Cerebrospinal Fluid-Contacting Neurons. Microscopy Research and Technique, 41, 57-83. http://dx.doi.org/10.1002/(SICI)1097-0029(19980401)41:1<57::AID-JEMT6>3.0.CO;2-R

[67] Vigh, B., et al. (2004) The System of Cerebrospinal Fluid-Contacting Neurons. Its Supposed Role in the Nonsynaptic Signal Transmission of the Brain. Histology and histopathology, 19, 607-628.

[68] Tozzi, A. and Peters, J.F. (2015) Brain Activity on Hypersphere. arXiv: 1512.00036 [q-bio.NC]. https://arxiv.org/ftp/arxiv/papers/1512/1512.00036.pdf

[69] Peters, J.F., Tozzi, A., Inan, E. and Ramanna, S. (2016) Entropy in Primary Sensory Areas Lower than in Associative Ones: The Brain Lies in Higher Dimensions than the Environment. BioRXiv, 071977. http://biorxiv.org/content/biorxiv/early/2016/08/29/071977.full.pdf

[70] Peters, J.F., Inan, E., Tozzi, A. and Ramanna, S. (2016) Primary Evidence of a Donut-Like, Fourth Spatial Dimension in the Brain. BioRXiv, 072397. http://biorxiv.org/content/biorxiv/early/2016/08/30/072397.full.pdf

[71] Tozzi, A. (2015) Information Processing in the CNS: A Supramolecular Chemistry? Cognitive Neurodynamics, 9, 463-477. http://dx.doi.org/10.1007/s11571-015-9337-1 http://arturotozzi.webnode.it/products/kindly-helped-by-karl-friston-information-processi ng-in-the-cns-a-supramolecular-chemistry-/

[72] Messori, C. (2004) Le Metamorfosi della Meraviglia. Riflessioni sui Percorsi della Conoscenza dall'Età del Bronzo ad Oggi. Maremmi Editori, Firenze Libri, Firenze. 
Submit or recommend next manuscript to OALib Journal and we will provide best service for you:

- Publication frequency: Monthly

- 9 subject areas of science, technology and medicine

- Fair and rigorous peer-review system

- Fast publication process

- Article promotion in various social networking sites (LinkedIn, Facebook, Twitter, etc.)

- Maximum dissemination of your research work

Submit Your Paper Online: Click Here to Submit

Or Contact service@oalib.com 